\title{
Efficient Local Map Search Algorithms for the Placement of Flying Relays
}

\author{
Junting Chen, Member, IEEE, and David Gesbert, Fellow, IEEE
}

\begin{abstract}
This paper studies the optimal unmanned aerial vehicle (UAV) placement problem for wireless networking. The UAV operates as a flying wireless relay to provide coverage extension for a base station (BS) and deliver capacity boost to a user shadowed by obstacles. While existing methods rely on statistical models for potential blockage of a direct propagation link, we propose an approach capable of leveraging local terrain information to offer performance guarantees. The proposed method allows to strike the best trade-off between minimizing propagation distances to ground terminals and discovering good propagation conditions. The algorithm only requires several propagation parameters, but it is capable to avoid deep propagation shadowing and is proven to find the globally optimal UAV position. Only a local exploration over the target area is required, and the maximum length of search trajectory is linear to the geographical scale. Hence, it lends itself to online search. Significant throughput gains are found when compared to other positioning approaches based on statistical propagation models.
\end{abstract}

\section{INTRODUCTION}

One significant challenge for wireless communication networks is the rapid increase of demand for high data rate and low latency wireless service. As a promising solution to future communication networks, substantial attention has been brought on the exploitation of UAVs as flying relays to connect BSs with the users in communication outage [1]-[7].

In UAV relaying over a dense urban environment, a fundamental challenge is the shadowing at the user side, where the degree of obstruction depends on the geographical environment. For example, the link between a UAV and a user may be in deep shadow when the UAV is located at the east side of a building, whereas, the propagation condition may be significantly improved when the UAV moves to the north side. It is difficult to know such fine-grained propagation condition prior to physically flying a UAV to a target position for assessment. Existing techniques that model the possible obstruction include specifying a larger path loss exponent, adding additional power loss, and constructing a random variable that describes the statistics of the shadowing. However, these models still over-simplify the actual propagation,

This paper has been accepted in IEEE Transactions on Wireless Communications. This research was funded, in part, by one or more of the following grants: No. ZDSYS201707251409055, No. 2017ZT07X152, No. 2018B030338001, No. 2018YFB1800800, and ERC under the European Union Horizon 2020 research and innovation program (Agreement no. 670896). This paper was presented, in part, at the IEEE International Conference on Communications, Paris, France, 2017.

Junting Chen is with the School of Science and Engineering and the Future Network of Intelligence Institute (FNii) at the Chinese University of Hong Kong, Shenzhen, Guangdong 518172, China (e-mail: juntingc@cuhk.edu.cn).

David Gesbert is with Department of Communication Systems, EURECOM, 06410 Sophia-Antipolis, France (e-mail: gesbert@eurecom.fr). because they implicitly assume that the degree of obstruction is homogeneous everywhere, meaning that the path loss is statistically the same everywhere given the same propagation distance (and elevation angle). Consequently, in a BS-UAVuser relay network, these models would predict the best UAV relay on the BS-user axis. In reality, however, a UAV may find significantly better propagation condition off the BS-user axis. We will demonstrate that substantial performance gain can be achieved when a more realistic fine-grained propagation model is exploited. The main goal of the paper is to develop an efficient and blockage-adaptive search strategy to explore finegrained propagation condition for the optimal UAV position.

\section{A. Related Works}

UAV optimization strategies are mainly developed based on specific air-to-ground path loss models. In [8]-[16], the path loss was modeled as a deterministic function of the UAV-to-user distance, irrelevant to specific UAV positions. Thanks to the simplicity of these distance-based models, [8][12] developed solutions to UAV navigation problems, [13] studied optimization strategies for multiple-input multipleoutput (MIMO) communications with UAVs with multiple antennas, and [14] optimized the UAV position for cooperative communications. The models used in $[8]-[16]$ imply that the path loss is the same under the same distance, but more detailed research in [17], [18] suggests that air-to-ground propagation should also depend on the elevation angle of the UAV-user link.

To capture the dependency of obstruction on elevation angles, in [17]-[21], the path loss was modeled as a random variable of the propagation distance and the elevation angle. Specifically, for the UAV position and coverage optimization problems studied in [18]-[20], the path loss was modeled as the average of the path loss under the LOS case and that under the NLOS case, where the larger the elevation angle, the higher the LOS probability. The work [21] considered the shadowing statistics as a function of elevation angle. Yet, these models implicitly assume that the degree of obstruction is homogeneous for the same distance and elevation angle. In practice, however, the degree of obstruction may vary from one location to another.

\section{B. Challenges and Our Contributions}

Although it is theoretically possible to associate each UAV and user position pair with a channel quality to capture the variation of the terrain, it is almost infeasible to implement a search strategy for the optimal UAV position. On one hand, it 
is time and energy prohibitive to maneuver a UAV for channel quality assessment at every possible position. On the other hand, even a 3D city map or a radio map can be constructed, it is computationally expensive to exhaustively search of the optimal UAV position. Moreover, the city map or radio map may not be updated or reliable in practice.

Therefore, it is essential to develop an air-to-ground path loss model to capture the variation of the terrain, and at the same time, consists of some nice structure that can be exploited to efficiently search for the optimal UAV position. Specifically, two main issues are to be addressed:

- How to model the air-to-ground path loss for the communication between ground users and low-altitude UAVs? We need a model that not only captures the various degrees of obstruction due to the complex terrain, but also facilitates a low complexity search strategy for optimal UAV positioning.

- How to plan an efficient search trajectory for the optimal UAV position? We desire to build a search path to find the globally optimal UAV position, while the maximum search length is only linear to the radius of the search area.

To answer these questions, we develop a nested segmented air-to-ground propagation model, which, for each user position, partitions the UAV search area into several segments, and associates each segment with a path loss model, such as LOS model, obstructed LOS model and non-line-of-sight (NLOS) model. This feature matches with the observation obtained from the experimental study in [22]. In addition, we impose that as the UAV moves away from the user, it can only enter propagation segments with a higher degree of obstruction, as shown in Fig. 1 (b). Such a requirement is consistent with many existing air-to-ground models from both academia and industry [17], [23], where the lower the elevation angle, the higher the probability for which the UAV-user link is obstructed. Using such a model, we develop a shaded-contourexploration algorithm to search for the globally optimal UAV position, and the algorithm only requires several propagation parameters but not the entire radio map. We prove that global optimum can be attained under a linear search trajectory. While this idea was partially exploited in our preliminary work [24], the work [24] focused only on the simplest case of two propagation segments and the optimality proof was not presented.

To summarize, the key contributions are made as follows:

- We propose a nested segmented propagation model to capture the fine-grained degree of obstruction in air-toground propagation.

- We develop a shaded-contour-exploration strategy to find the optimal UAV position for a single user relay system, with proven global optimality and linear search complexity. Substantial performance gain for the case of clustered multiple users is also numerically demonstrated.

- We perform numerical experiments to evaluate the performance of the UAV relay system and compare with existing approaches from existing models. Substantial throughput gain is found from a simulated Manhattan- like urban environment.

The rest of the paper is organized as follows. Section II establishes the nested segmented air-to-ground propagation model. Section III develops the search algorithm, and Section IV] establishes theoretical results on the global optimality. $\mathrm{Nu}-$ merical results are demonstrated in Section $\mathrm{V}$, and conclusions are given in Section VI

\section{SySTEM MODEL}

Consider a cellular network in an urban environment, where the $\mathrm{BS}$ is placed on rooftop or on a tower that is higher than all the buildings. Due to the possibly dense distribution of buildings and vegetation, it is likely that signals transmitted from the BS are significantly obstructed from users on street levels. Consider to deploy a UAV as a flying relay that connects a user with the BS. Assume that the UAV moves at a fixed height $H_{\mathrm{d}}>H_{\mathrm{b}}$, where $H_{\mathrm{b}}$ is the height of the BS. Denote the horizontal positions of the UAV, BS, and user, respectively, as $\mathbf{x}, \mathbf{x}_{\mathrm{b}}, \mathbf{x}_{\mathrm{u}} \in \mathbb{R}^{2}$, and hence, $\left(\mathbf{x}, H_{\mathrm{d}}\right),\left(\mathbf{x}_{\mathrm{b}}, H_{\mathrm{b}}\right),\left(\mathbf{x}_{\mathrm{u}}, 0\right) \in \mathbb{R}^{3}$ are, respectively, the positions of the UAV, BS, and user in 3D.

Note that the signal from the UAV can still be obstructed from the user due to local obstacles surrounding the user. On the other hand, the UAV relay cannot move too close to the user because it needs to balance the relay link with the BS. To address this dilemma, the goal of this paper is to optimize the horizontal position $\mathrm{x} \in \mathbb{R}^{2}$ for the UAV.

\section{A. Channel Model for the UAV-BS Link}

Define the channel as the deterministic power gain averaged over small scale fading. The BS-UAV channel is modeled as

$$
g_{\mathrm{b}}(\mathbf{x})=\beta_{0} d_{\mathrm{b}}(\mathbf{x})^{-\alpha_{0}}
$$

where $d_{\mathrm{b}}(\mathbf{x})=\sqrt{\left\|\mathbf{x}-\mathbf{x}_{\mathrm{b}}\right\|^{2}+\left(H_{\mathrm{d}}-H_{\mathrm{b}}\right)^{2}}$ is the distance from the UAV at $\left(\mathbf{x}, H_{\mathrm{d}}\right)$ to the $\mathrm{BS}$ at $\left(\mathbf{x}_{\mathrm{b}}, H_{\mathrm{b}}\right)$, and the constants $\alpha_{0}>1$ and $\beta_{0}>0$ are the classical path loss exponent and offset parameters. Such a model corresponds to the common scenario where the altitudes $H_{\mathrm{b}}$ of the BS and $H_{\mathrm{d}}$ of the UAV are large enough such that there is always a LOS condition for the BS-UAV link. We thus focus on modeling the UAV-user link in Section II-B.

\section{B. Channel Model for the UAV-user Link: A Nested Segmented Model}

In the classical large-scale fading channel model, the channel gain is modeled as $G_{\mathrm{dB}}=b-a \log _{10} d+\xi$, where $\xi$ is a random variable to capture the shadowing effect. Inspired by the geometry in the ray-tracing propagation modeling as illustrated in Fig. 1, we propose to split $G_{\mathrm{dB}}$ into several components each associated with a set of parameters $a, b$ and a random component $\xi_{k}$ for a specific degree of link obstruction.

Specifically, let $\mathbb{D} \subseteq \mathbb{R}^{2}$ be the domain of all possible UAV positions $\mathbf{x}$ at constant altitude $H_{\mathrm{d}}$. Consider a partition of $\mathbb{D}$ into $K$ disjoint segments $\mathbb{D}=\mathcal{D}_{1}\left(\tilde{\mathbf{x}}_{\mathrm{u}}\right) \cup \mathcal{D}_{2}\left(\tilde{\mathbf{x}}_{\mathrm{u}}\right) \cup \cdots \cup \mathcal{D}_{K}\left(\tilde{\mathbf{x}}_{\mathrm{u}}\right)$, where $\mathcal{D}_{k} \cap \mathcal{D}_{j}=\varnothing$, for $k \neq j$, and $\mathcal{D}_{k}\left(\tilde{\mathbf{x}}_{\mathrm{u}}\right)$ denotes the region of UAV locations for which the UAV maintains a degree- $k$ 


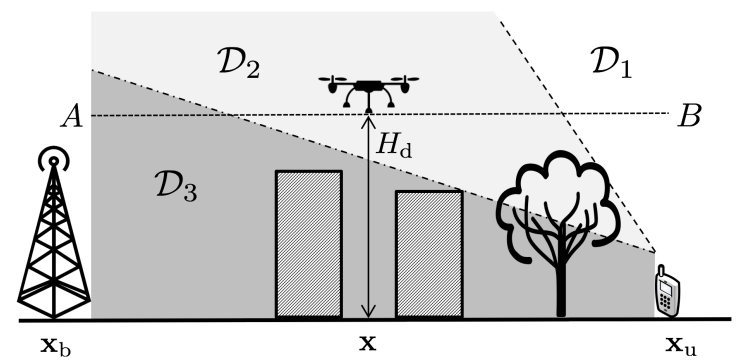

(a) Horizontal view

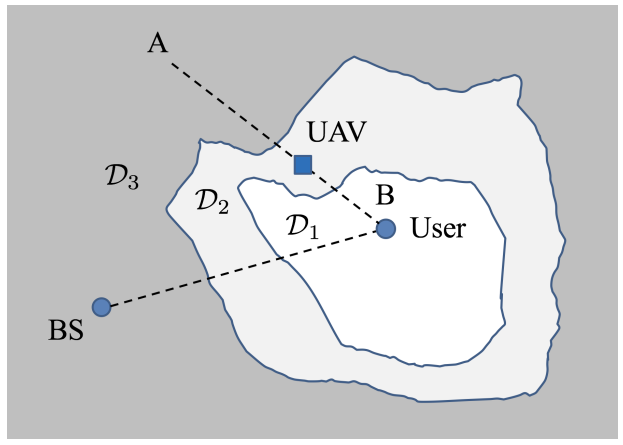

(b) Nested segmented model

Figure 1. A geometric interpretation of the nested segmented model from (a) horizontal view and (b) top view.

of LOS obstruction from the user. The proposed segmented propagation model for $G_{\mathrm{dB}}$ is specified as:

$$
G_{\mathrm{dB}}(\mathbf{x})=\sum_{k=1}^{K}\left(b_{k}-a_{k} \log _{10} d_{\mathrm{u}}(\mathbf{x})+\xi_{k}\right) \mathbb{I}\left\{\mathbf{x} \in \mathcal{D}_{k}\right\}
$$

where $d_{\mathrm{u}}(\mathbf{x})=\sqrt{\left\|\mathbf{x}-\mathbf{x}_{\mathrm{u}}\right\|^{2}+\left(H_{\mathrm{d}}-H_{\mathrm{u}}\right)^{2}}$ is the distance from the UAV located at $\left(\mathbf{x}, H_{\mathrm{d}}\right)$ to the user at $\tilde{\mathbf{x}}_{\mathrm{u}}=\left(\mathbf{x}_{\mathrm{u}}, H_{\mathrm{u}}\right)$, $a_{k}$ and $b_{k}$ are some parameters, and $\mathbb{I}\{A\}$ is an indicator function taking value 1 if condition $A$ is satisfied, and 0 otherwise. The random variable $\xi_{k}$ captures the residual shadowing effect. The segment parameters $\left\{\alpha_{k}, \beta_{k}, \mathcal{D}_{k}\right\}$ are assumed to satisfy the following conditions:

1) The propagation segment $\mathcal{D}_{k}$ exhibits a higher degree of LOS obstruction than $\mathcal{D}_{k-1}$, i.e., for $k=2,3, \ldots, K$ and any UAV position $\mathbf{x}$,

$$
b_{k}-a_{k} \log _{10} d_{\mathrm{u}}(\mathbf{x})<b_{k-1}-a_{k-1} \log _{10} d_{\mathrm{u}}(\mathbf{x})
$$

or, in the linear scale representation,

$$
\beta_{k} d_{\mathrm{u}}(\mathbf{x})^{-\alpha_{k}}<\beta_{k-1} d_{\mathbf{u}}(\mathbf{x})^{-\alpha_{k-1}}
$$

where $b_{k}=10 \log _{10} \beta_{k}$ and $a_{k}=10 \alpha_{k}$.

2) The propagation segments $\mathcal{D}_{k}$ are nested along any directions from the user, i.e., for any $\mathbf{x} \in \mathcal{D}_{k}$ and $0 \leq \rho \leq 1$

$$
\mathbf{x}_{\mathrm{u}}+\rho\left(\mathbf{x}-\mathbf{x}_{\mathrm{u}}\right) \in \mathcal{D}_{j}, \quad \text { for some } j \leq k .
$$

In other words, when the UAV moves towards the user, the UAV-user channel tends to become always less obstructed [25] as there are fewer obstacles in between the UAV and the user, as illustrated in Fig. 1 .
It is intuitive that if the parameters $\left\{\alpha_{k}, \beta_{k}, \mathcal{D}_{k}\right\}$ are obtained correctly, the variance of the residual term $\xi_{k}$ can be substantially reduced compared to that of the random component $\xi$ in the classical channel model. For example, the variance of the shadowing in the LOS case is believed to be much smaller than that in the combined LOS and NLOS case.

With such an insight, we focus on optimizing the UAV network based on the average channel gain $\bar{G}_{\mathrm{dB}}(\mathbf{x}) \triangleq$ $\mathbb{E}\left\{G_{\mathrm{dB}}(\mathbf{x}) \mid\left\{\mathcal{D}_{k}\right\}\right\}$ given the propagation segments, where the expectation is taken over $\xi_{k}$. Therefore, assuming $\xi_{k}$ with zero mean, the deterministic channel in linear scale $10 \log _{10} g_{\mathrm{u}}(\mathbf{x}) \triangleq \bar{G}_{\mathrm{dB}}(\mathbf{x})$ can be written as:

$$
g_{\mathrm{u}}(\mathbf{x})=\sum_{k=1}^{K} \beta_{k} d_{\mathrm{u}}(\mathbf{x})^{-\alpha_{k}} \mathbb{I}\left\{\mathbf{x} \in \mathcal{D}_{k}\right\}
$$

The problem of learning $\alpha_{k}, \beta_{k}$, and the statistics of $\xi_{k}$ from measurement data has been partially attempted in [26], [27], and it is not the focus of this paper. Here, we assume that $\alpha_{k}$, $\beta_{k}$, and the statistics of $\xi_{k}$ are perfectly known, but we still need to (partially) determine $\mathcal{D}_{k}$, i.e., the boundaries shown in Fig. 1 (b), to help search for the optimal UAV position. Note that learning the entire $\mathcal{D}_{k}$ is much more difficult and time consuming (or even prohibitive) than learning $\alpha_{k}$ and $\beta_{k}$. The goal of this paper is to optimize the UAV position by only partially exploring $\mathcal{D}_{k}$.

\section{Problem Formulation and Application Examples}

Consider an objective cost function $f\left(g_{\mathrm{u}}, g_{\mathrm{b}}\right)$ of the UAVuser channel gain $g_{\mathrm{u}}$ and the BS-UAV channel gain $g_{\mathrm{b}}$. Assume that $f(x, y)$ is a continuous and decreasing function in $x$ and $y$, respectively. A generic UAV positioning problem can be formulated as follow 1

$$
\mathscr{P}: \quad \underset{\mathbf{x} \in \mathbb{R}^{2}}{\operatorname{minimize}} f\left(g_{\mathrm{u}}(\mathbf{x}), g_{\mathrm{b}}(\mathbf{x})\right) .
$$

The problem formulation $\mathscr{P}$ can capture many applications for a variety of relay transmission strategies. Three examples are illustrated as follows, where we choose some simple formulations for easy demonstration of the proposed algorithm.

Consider that the transmission from the BS to the UAV is modeled as $y_{\mathrm{r}}=\sqrt{P_{\mathrm{b}} g_{\mathrm{b}}} a_{\mathrm{b}} s+n_{\mathrm{r}}$, and that from the UAV to the user is modeled as $y_{\mathrm{u}}=\sqrt{P_{\mathrm{u}} g_{\mathrm{u}}} a_{\mathrm{u}} s_{\mathrm{r}}+n_{\mathrm{u}}$, where $n_{\mathrm{r}}, n_{\mathrm{u}} \sim \mathcal{C N}(0,1)$ are the receive noise at the UAV relay and the user, respectively, $s, s_{\mathrm{r}} \sim \mathcal{N}(0,1)$ are the transmit signals from the BS and the UAV relay, respectively, and $P_{\mathrm{u}}$ and $P_{\mathrm{b}}$ are transmission powers at the UAV and the BS, respectively. The variables $a_{\mathrm{b}}$ and $a_{\mathrm{u}}$ model the small scale fading on the BS-UAV link and the UAV-user link, respectively. For Rayleigh fading channels, $\left|a_{\mathrm{b}}\right|^{2}$ and $\left|a_{\mathrm{u}}\right|^{2}$ are assumed to follow exponential distribution with parameter (normalized as) $\lambda=1$.

1) Amplify-and-Forward: In amplify-and-forward relaying, the UAV relays the information by transmitting $s_{\mathrm{r}}=$ $y_{\mathrm{r}} / \sqrt{P_{\mathrm{b}} g_{\mathrm{b}}\left|a_{\mathrm{r}}\right|^{2}+1}$, where the scaling factor $\sqrt{P_{\mathrm{b}} g_{\mathrm{b}}\left|a_{\mathrm{r}}\right|^{2}+1}$

\footnotetext{
${ }^{1}$ More generally, one can design the cost function $f$ in terms of the UAV position $\mathbf{x}$ to capture other system characteristics, such as antenna pattern and effect of small-scale fading.
} 


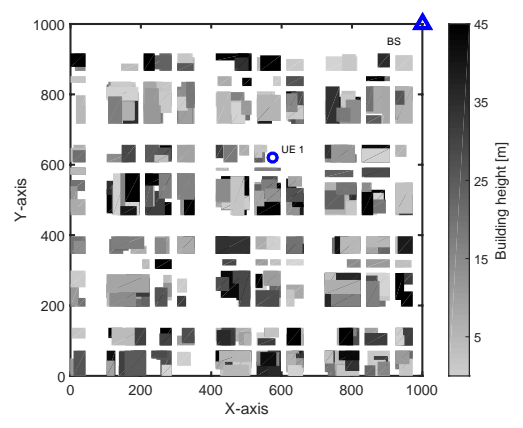

(a) Urban topology

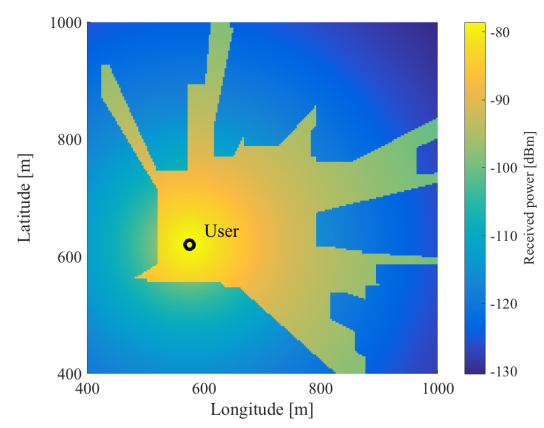

(b) Power map

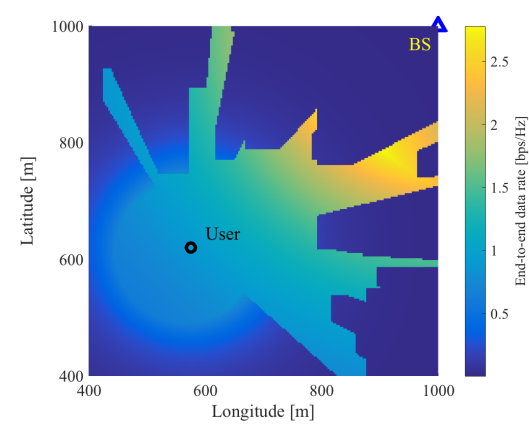

(c) Capacity map

Figure 2. (a) A map of a dense urban area, where the rectangles denote the building with colors representing their heights. (b) The simulated received power map corresponding to every UAV position. (c) The simulated end-to-end capacity map.

is to normalize the transmission power at the UAV to be $P_{\mathrm{u}}$. It was shown in [28], [29] that the capacity of the relay channel is given by $C_{\mathrm{AF}}=\frac{1}{2} \log _{2}\left(1+q\left(P_{\mathrm{b}} g_{\mathrm{b}}\left|a_{\mathrm{r}}\right|^{2}, P_{\mathrm{u}} g_{\mathrm{u}}\left|a_{\mathrm{u}}\right|^{2}\right)\right)$, where $q(x, y) \triangleq x y /(x+y+1)$ and the parameter $\frac{1}{2}$ is to capture the fact that the information requires two time slots to reach the user. The outage probability with respect to a target data rate $R$ was shown to be [29. Lemma 1] $\mathbb{P}\left\{C_{\mathrm{AF}}<R\right\} \approx\left(\frac{1}{P_{\mathrm{b}} g_{\mathrm{b}}}+\frac{1}{P_{\mathrm{u}} g_{\mathrm{u}}}\right)\left(2^{2 R}-1\right)^{2}$ under high signalto-noise ratio (SNR), i.e., $P_{\mathrm{b}} g_{\mathrm{b}}, P_{\mathrm{u}} g_{\mathrm{u}} \gg 12$

Therefore, to minimize the outage probability of the relay channel, the desired UAV position can be determined as the solution to $\mathscr{P}$ with a cost function given as:

$$
f\left(g_{\mathrm{u}}(\mathbf{x}), g_{\mathrm{b}}(\mathbf{x})\right):=\frac{1}{P_{\mathrm{u}} g_{\mathrm{u}}(\mathbf{x})}+\frac{1}{P_{\mathrm{b}} g_{\mathrm{b}}(\mathbf{x})} .
$$

2) Decode-and-Forward: In decode-and-forward relaying, the UAV fully decodes the message $\hat{s}$ from the receive signal $y_{\mathrm{r}}$, and transmits $s_{\mathrm{r}}=\hat{s}$ to the user. The maximum capacity of such a decode-and-forward relay system can be shown to be $C_{\mathrm{DF}}=\frac{1}{2} \min \left\{\log _{2}\left(1+P_{\mathrm{b}} g_{b}\left|a_{b}\right|^{2}\right), \log _{2}\left(1+P_{\mathrm{u}} g_{\mathrm{u}}\left|a_{\mathrm{u}}\right|^{2}\right)\right\}[29]$, [30]. Using Jensen's inequality $\mathbb{E}\{C(x)\} \leq C(\mathbb{E}\{x\})$ on a concave function $C(x)$, an upper bound of the ergodic capacity $\mathbb{E}\left\{C_{\mathrm{DF}}\right\}$ is given by $\frac{1}{2} \min \left\{\log _{2}\left(1+P_{\mathrm{b}} g_{b}\right), \log _{2}\left(1+P_{\mathrm{u}} g_{\mathrm{u}}\right)\right\}$. The desired UAV position can be determined by maximizing such a capacity bound. Equivalently, the problem $\mathscr{P}$ can be specified by choosing the following cost function:

$$
\begin{aligned}
f\left(g_{\mathrm{u}}(\mathbf{x}), g_{\mathrm{b}}(\mathbf{x})\right):=\max \{ & -\log _{2}\left(1+P_{\mathrm{b}} g_{b}(\mathbf{x})\right), \\
& \left.-\log _{2}\left(1+P_{\mathrm{u}} g_{\mathrm{u}}(\mathbf{x})\right)\right\} .
\end{aligned}
$$

A numerical example is given in Fig. 2, where Fig. 2(b) simulates the received power of the UAV-user signal with respect to (w.r.t.) every UAV position under a segmented propagation model with $K=2$ segments. Fig. 2 (c) shows the corresponding relay channel capacity from the BS to the user via the UAV. It is not trivial to find the optimal UAV relay position due to the irregular propagation pattern.

\footnotetext{
${ }^{2}$ The original problem in [29] considered a diversity scheme that combines the signal from the relay and the signal from the BS. Such a strategy also leads to problem $\mathscr{P} 1$ under high SNR
}

3) Multiuser Clustered around a Hotspot: Suppose that there are $N_{\mathrm{u}}$ users clustered around a hotspot centered at $\mathbf{x}_{\mathrm{c}}$ with radius $r_{\mathrm{u}}$. Let $f^{(i)}(\mathbf{x})$ be the cost function taking the form in 77 for the $i$ th user located at position $\mathbf{x}_{\mathrm{u}}^{(i)}$. Specifically, the UAV-user gain $g_{\mathrm{u}}\left(\mathbf{x} ; \mathbf{x}_{\mathrm{u}}^{(i)}\right)$ in 5 ) is computed based on the user position $\mathbf{x}_{i}$. Consider to maximize the sum rate $-\frac{1}{N_{\mathrm{u}}} \sum_{i=1}^{N_{\mathrm{u}}} f^{(i)}(\mathbf{x})$. One may consider to simplify and approximate the cost function $\bar{f}(\mathbf{x}) \triangleq \frac{1}{N_{\mathrm{u}}} \sum_{i=1}^{N_{\mathrm{u}}} f^{(i)}(\mathbf{x})$ by constructing a virtual user indexed as $i=N_{\mathrm{u}}+1$. The virtual user is virtually placed at the hotspot center $\mathbf{x}_{\mathrm{c}}$ (as a similar topological model discussed in [15]), and the corresponding channel gain $g_{\mathrm{u}}\left(\mathbf{x} ; \mathbf{x}_{\mathrm{c}}\right)$ for is modeled using the segmented log-distance model (5), except that the propagation segment depends on the majority vote from the $N_{\mathrm{u}}$ actual users. Specifically, the UAV position $\mathbf{x}$ belongs to the $k$ th propagation segment $\tilde{\mathcal{D}}_{k}\left(\mathbf{x}_{\mathrm{c}}\right)$ (for the virtual user), if the majority UAV-user links $\left(\mathbf{x}, \mathbf{x}_{\mathrm{u}}^{(i)}\right)$ belong to the $k$ the segment. As a result, it is clear that the cost function $f(\mathbf{x}) \triangleq f^{\left(N_{\mathrm{u}}+1\right)}(\mathbf{x})$ for the virtual user is a good approximation of the average cost $\frac{1}{N_{\mathrm{u}}} \sum_{i=1}^{N_{\mathrm{u}}} f^{(i)}(\mathbf{x})$ as long as the cluster radius $r_{\mathrm{u}}$ is small.

While such an approximation is only suboptimal, in Section $\mathrm{V}-\mathrm{B}$ we numerically demonstrate that our proposed strategy that solves $\mathscr{P}$ still provide reasonably good performance up to moderate cluster radius $r_{\mathrm{u}}$ as compared to stochastic optimization using simplified models.

\section{AlgorithM DESIGNS}

In this section, we first derive some useful insights on the optimal UAV positions, and then develop a polar representation tool. Based on that, we develop the search algorithm for the optimal UAV position.

\section{A. Properties of the Optimal UAV Position}

Proposition 1. The optimal solution $\mathrm{x}^{*}$ to $\mathscr{P}$ is either on the $B S$-user axis, or on the boundary between two propagation segments.

Proof. Suppose there is a solution $\mathbf{x}$ which is strictly inside a propagation segment $\mathcal{D}_{k}$ and is off the BS-user axis. Then there exists a direction $\delta$, such that for a sufficiently small $\epsilon>0$, the new UAV position $\mathbf{x}+\epsilon \boldsymbol{\delta}$ decreases the distances to 


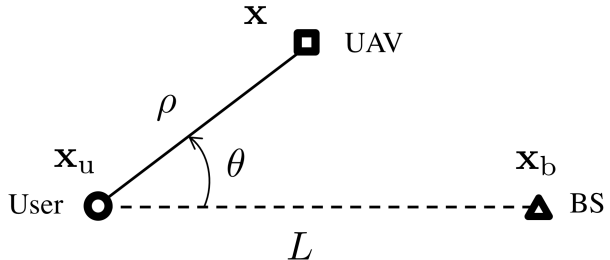

Figure 3. Illustration of a polar representation of the UAV position $\mathbf{x}$.

both the user and the BS, while at the same time, $\mathbf{x}+\epsilon \boldsymbol{\delta} \in \mathcal{D}_{k}$. From the nested segmented model (5) and also the UAV-BS channel model (1), smaller distances $d_{\mathrm{u}}$ and $d_{\mathrm{b}}$ imply larger channel gains $g_{\mathrm{u}}$ and $g_{\mathrm{b}}$. Due to the monotonicity property of the cost function $f\left(g_{\mathrm{u}}, g_{\mathrm{b}}\right)$, larger channel gains yield a smaller cost value, which implies that $\mathbf{x}$ is not the optimal solution. By contradiction, the proposition is therefore confirmed.

However, it is still costly to search along the segment boundaries because the boundaries may have complex shapes, resulting in an unacceptably long trajectory (which could be super-linear to the radius of the search area) for the UAV as demonstrated in Fig. 2

\section{B. Polar Representation}

To develop a more efficient search strategy, we transform the problem into the one expressed over a polar coordinate system.

Let $\rho=\left\|\mathbf{x}-\mathbf{x}_{\mathbf{u}}\right\|$ be the ground projected distance from the user at $\left(\mathbf{x}_{\mathrm{u}}, H_{\mathrm{u}}\right)$ to the UAV at $\left(\mathbf{x}, H_{\mathrm{d}}\right)$. Let $\theta \in(-\pi, \pi)$ be the deviation angle from the user-to-BS direction to the user-to-UAV direction as illustrated in Fig. 3 Denote $\mathbf{u}=$ $\left(u_{1}, u_{2}\right) \triangleq \frac{\mathbf{x}_{b}-\mathbf{x}_{u}}{\left\|\mathbf{x}_{b}-\mathbf{x}_{u}\right\|}$ as the normalized user-to-BS direction. The UAV position $\mathbf{x} \in \mathbb{R}^{2}$ can be equivalently expressed by $(\rho, \theta)$ as

$$
\mathbf{x}(\rho, \theta)=\mathbf{x}_{\mathbf{u}}+\rho \mathbf{M}(\theta) \mathbf{u}
$$

where

$$
\mathbf{M}(\theta)=\left[\begin{array}{ll}
\cos \theta & -\sin \theta \\
\sin \theta & \cos \theta
\end{array}\right]
$$

is a rotation matrix, and

$$
\theta=\operatorname{sign}\left(z_{2} u_{1}-z_{1} u_{2}\right) \cdot \arccos \left(\mathbf{z}^{\mathrm{T}} \mathbf{u} / \rho\right)
$$

in which $\mathbf{z}=\left(z_{1}, z_{2}\right) \triangleq \mathbf{x}-\mathbf{x}_{\mathrm{u}}, \operatorname{sign}(x)=1$ if $x>0$, and $\operatorname{sign}(x)=-1$, otherwise.

We now define an alternative expression for the cost function $f\left(g_{\mathrm{u}}(\mathbf{x}), g_{\mathrm{b}}(\mathbf{x})\right)$.

Definition 1 (Fictitious Segment Cost Function). For $\mathbf{x}(\rho, \theta) \in \mathcal{D}_{k}, \quad k=1,2, \ldots, K$, the cost function $f\left(g_{\mathrm{u}}(\mathbf{x}), g_{\mathrm{b}}(\mathbf{x})\right)$ can be written as

$$
f\left(g_{\mathrm{u}}(\mathbf{x}), g_{\mathrm{b}}(\mathbf{x})\right)=F_{k}(\rho, \theta)
$$

where

$$
F_{k}(\rho, \theta) \triangleq f\left(g_{\mathrm{u}}^{(k)}(\mathbf{x}(\rho, \theta)), g_{\mathrm{b}}(\mathbf{x}(\rho, \theta))\right)
$$

and $g_{\mathbf{u}}^{(k)}(\mathbf{x}) \triangleq \beta_{k} d_{\mathbf{u}}(\mathbf{x})^{-\alpha_{k}}$ is the UAV-user channel in the $k$ th segment from the channel model (5).
As a result, the objective function in $\mathscr{P}$ is transformed into the polar domain as $F(\rho, \theta)=\sum_{k=1}^{K} F_{k}(\rho, \theta) \mathbb{I}\{\mathbf{x}(\rho, \theta) \in$ $\left.\mathcal{D}_{k}\right\}$.

The motivation of working on the polar domain is that by fixing the deviation $\theta$, increasing $\rho$ only worsens the propagation condition according to the nested property (3)-(4). In addition, the overall cost function $F(\rho, \theta)$ is discontinuous because it contains the indicator functions, but the functions $F_{k}(\rho, \theta)$ are continuous. As result, the functions $F_{k}(\rho, \theta)$ can be used to derive search trajectories.

\section{Search Trajectory Design for $K=2$}

The algorithm is better illustrated starting from the two segment case, where $\mathcal{D}_{1}$ corresponds to the LOS segment and $\mathcal{D}_{2}$ corresponds to the NLOS segment.

1) Search on the BS-user Axis: Let the UAV start from the BS. It first moves towards the user until it finds two critical positions (if they exist) $\mathbf{x}_{k}^{0}=\mathbf{x}\left(\rho_{k}^{0}, 0\right), k=1,2$, which correspond to the points achieving the minimum cost over the BS-user axis in the LOS region and NLOS region, respectively. Specifically, the parameters $\rho_{k}^{0}$ are the solutions that minimize the fictitious cost $F_{k}(\rho, 0)$ along the BS-user axis $\mathbf{x}(\rho, 0) \in \mathcal{D}_{k}$, for $k=1,2$, in the two segment case.

For example, when the UAV is initially in the NLOS region, it can move up to the LOS-NLOS boundary (see Section III-E for a discussion on the detection method); with that, it can solve for $\rho_{1}^{0}$ and $\rho_{2}^{0}$ to obtain the critical points $\mathbf{x}_{1}^{0}$ and $\mathbf{x}_{2}^{0}$. On the other hand, when the UAV is initially in the LOS region, it can compute the critical position $\mathbf{x}_{1}^{0}$ in the LOS region, while $\mathbf{x}_{2}^{0}$ does not exist.

2) Search on the Right Branch: Starting from the critical position $\mathrm{x}_{1}^{0}$ which minimizes the cost function on the LOS portion of the BS-user axis, the UAV first moves to $\mathbf{x}\left(\rho_{1}^{0}, \delta / \rho_{1}^{0}\right)$, i.e., a position just on the right of $\mathbf{x}_{1}^{0}$ in Fig. 4 (a), where $\delta$ is a chosen step size. It then proceeds according to the following two phases; at the same time, it keeps the track record of the minimum cost value $F_{\min }$ discovered and the corresponding position $\hat{\mathbf{x}}(\hat{\rho}, \hat{\theta})$ that achieves $F_{\min }=F(\hat{\rho}, \hat{\theta})$ :

- If the UAV is in the LOS region, it moves away from the user. Specifically, it moves from $\mathbf{x}(\rho, \theta)$ to $\mathbf{x}(\rho+\delta, \theta)$.

- If the UAV is in the NLOS region, it moves in the direction that maintains the same fictitious cost $F_{1}(\rho, \theta)$ as it were in the LOS region, i.e., contour of $F_{1}(\rho, \theta)=C$ specified by

$$
\frac{\partial F_{1}(\rho, \theta)}{\partial \rho} d \rho+\frac{\partial F_{1}(\rho, \theta)}{\partial \theta} d \theta=0 .
$$

It can be shown that (Lemma 2 in Appendix C), $\partial F_{1}(\rho, \theta) / \partial \theta \neq 0$ for $\theta \neq 0$. As a result from (8), we have

$$
d \mathbf{x}=\mathbf{M}(\theta) \mathbf{u} d \rho+\rho \frac{d}{d \theta} \mathbf{M}(\theta) \mathbf{u}\left(-\frac{\partial F_{1}}{\partial \theta}\right)^{-1} \frac{\partial F_{1}}{\partial \rho} d \rho .
$$

Thus, the UAV updates its position from $\mathrm{x}$ to $\mathrm{x}+\Delta \mathrm{x}$, where

$$
\Delta \mathbf{x}=\gamma\left[\mathbf{M}(\theta) \mathbf{u}+\rho \frac{d}{d \theta} \mathbf{M}(\theta) \mathbf{u}\left(-\frac{\partial F_{1}}{\partial \theta}\right)^{-1} \frac{\partial F_{1}}{\partial \rho}\right]
$$

${ }^{3}$ For mathematical completeness, the partial derivative is defined as $\frac{\partial f\left(x_{0}, y_{0}\right)}{\partial x}=\lim _{t \uparrow 0} \frac{1}{t}\left[f\left(x_{0}+t, y_{0}\right)-f\left(x_{0}, y_{0}\right)\right]$ throughout this paper. 


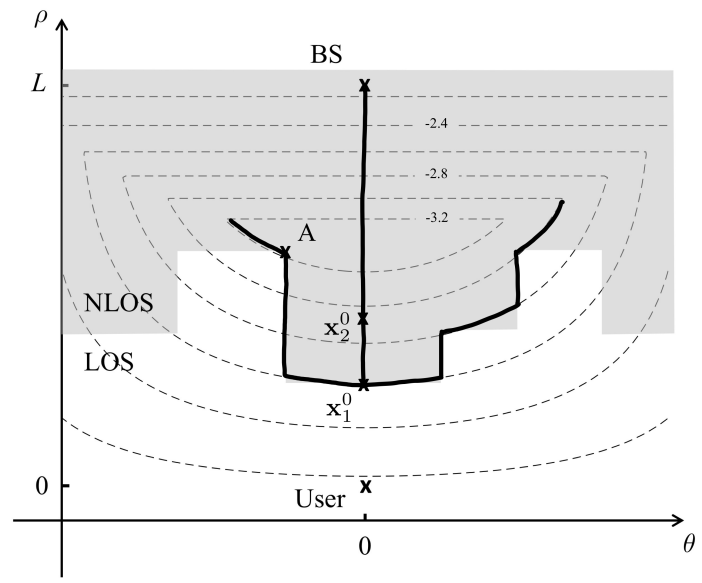

(a) A search path (solid black) shown in the polar domain

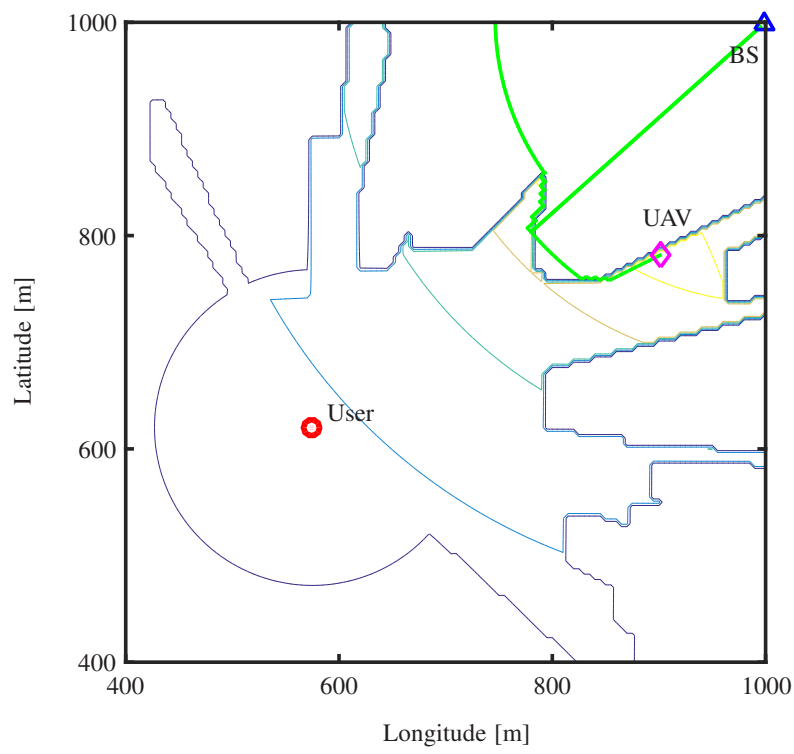

(b) A search path (green) shown in the Euclidean domain

Figure 4. (a) An example UAV search trajectory under two propagation segment case in the polar domain. The dashed lines represent the contours of the fictitious cost function $F_{1}(\rho, \theta)=C$. (b) A UAV search path (greed lines) in the Euclidean domain for the topology in Fig. 2 (a). The curves in other colors represent the contours of the cost function (7), and the purple diamond represents the globally optimal position.

in which, $\gamma>0$ is chosen such that the step size $\|\Delta \mathbf{x}\|=$ $\delta$ and the UAV moves in the direction away from the user (see Fig. 4).

The search at this branch is completed whenever the UAV reaches a point $\mathbf{x}(\rho, \theta)$ such that either $\partial F_{1}(\rho, \theta) / \partial \rho \geq 0$ or $\rho \geq L \cos \theta$, where $L \triangleq\left\|\mathbf{x}_{\mathrm{b}}-\mathbf{x}_{\mathrm{u}}\right\|$. The justification of the two stopping criteria will become clear in Section IV-A

3) Search on the Left Branch: Starting from the critical position $\mathrm{x}_{1}^{0}$, the $\mathrm{UAV}$ moves to $\mathrm{x}\left(\rho_{1}^{0},-\delta / \rho_{1}^{0}\right)$, i.e., a position just on the left of $x_{1}^{0}$ in Fig. 4 (a). It repeats the same process as in Section III-C2 until it meets the stopping criteria. When the search is completed, the track record $\hat{\mathrm{x}}$ that achieves the least cost will be the desired UAV position. Note that the position $\hat{\mathbf{x}}$, for example, point A in Fig. 4 (a), is not necessarily where the search terminates.

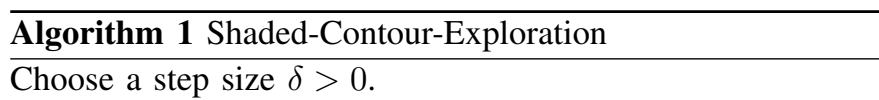

1) Search on the BS-user axis: Find the critical points $\rho_{k}^{0}, k=1,2, \ldots, K$, defined in 14 . Initialize $F_{\min }=$ $F_{1}\left(\rho_{1}^{0}, 0\right)$ and $\hat{\mathbf{x}}=\mathbf{x}\left(\rho_{1}^{0}, 0\right)$, where $\mathbf{x}(\rho, \theta)$ is defined in (8). Initialize $k=1$.

2) Search on the right branch: Set $\mathbf{x} \leftarrow \mathbf{x}\left(\rho_{k}^{0}, \delta / \rho_{k}^{0}\right)$.

3) For each iterate $\mathbf{x}$, compute $\rho=\left\|\mathbf{x}-\mathbf{x}_{\mathrm{u}}\right\|$ and $\theta$ from (10).

a) Search in the virtual LOS region: If $x \in$ $\bigcup_{j=1}^{k} \mathcal{D}_{j}$, update

$$
\mathbf{x} \leftarrow \mathbf{x}(\rho+\delta, \theta) .
$$

When $F(\rho+\delta, \theta)<F_{\min }$, update the record $F_{\min } \leftarrow F(\rho+\delta, \theta)$ and $\hat{\mathbf{x}} \leftarrow \mathbf{x}(\rho+\delta, \theta)$.

b) Search in the virtual NLOS region: If $\mathrm{x} \notin$ $\bigcup_{j=1}^{k} \mathcal{D}_{j}$, update $\mathrm{x} \leftarrow \mathbf{x}+\Delta \mathbf{x}$, where

$$
\Delta \mathbf{x}=\gamma\left[\mathbf{M}(\theta) \mathbf{u}+\rho \frac{d}{d \theta} \mathbf{M}(\theta) \mathbf{u}\left(-\frac{\partial F_{k}}{\partial \theta}\right)^{-1} \frac{\partial F_{k}}{\partial \rho}\right]
$$

where $\gamma>0$ is chosen such that $\|\Delta \mathbf{x}\|=\delta$.

Repeat this step until either (i) $\rho \geq L \cos \theta$ or (ii) $\partial F_{k}(\rho, \theta) / \partial \rho \geq 0$.

4) Search on the left branch: Set $\mathbf{x} \leftarrow \mathbf{x}\left(\rho_{k}^{0},-\delta / \rho_{k}^{0}\right)$. Repeat Step 3.

5) Let $k \leftarrow k+1$. Repeat from Step 2 until $k>K-1$.

6) If $F_{K}\left(\rho_{K}^{0}, 0\right)<F_{\min }$, then $F_{\min } \leftarrow F_{K}\left(\rho_{K}^{0}, 0\right)$ and $\hat{\mathbf{x}} \leftarrow \mathbf{x}\left(\rho_{K}^{0}, 0\right)$.

\section{Search Trajectory Design for Arbitrary $K$}

In the case of more than two propagation segments, one can generate $K-1$ search trajectories following a similar procedure in Section III-C, where each trajectory is computed by partitioning the whole area into two virtual propagation regions: virtual LOS region and virtual NLOS region. Specifically, for the $k$ th search trajectory, the virtual LOS region is defined by grouping the first $k$ propagation segments together $\tilde{\mathcal{D}}_{k} \triangleq \bigcup_{j=1}^{k} \mathcal{D}_{j}$, and correspondingly, the virtual NLOS region is defined as $\tilde{\mathcal{D}}_{k}^{\mathrm{c}} \triangleq \mathbb{D} \backslash \tilde{\mathcal{D}}_{k}=\bigcup_{j=k+1}^{K} \mathcal{D}_{j}$. The whole algorithm can be summarized as follows.

- Search on BS-user axis: Let the UAV start from the BS. It first moves towards the user along the BS-user axis until it finds the $K-1$ critical positions $\mathbf{x}_{k}^{0}=\mathbf{x}\left(\rho_{k}^{0}, 0\right)$, $k=1,2, \ldots, K-1$, each of which corresponds to a point achieving the minimum cost over the BS-user axis in a virtual LOS region, i.e., $\rho_{k}^{0}$ are the solutions to

$$
\begin{array}{ll}
\underset{\rho \geq 0}{\operatorname{minimize}} & F_{k}(\rho, 0) \\
\text { subject to } & \mathbf{x}(\rho, 0) \in \bigcup_{j=1}^{k} \mathcal{D}_{j}
\end{array}
$$

for $k=1,2, \ldots, K-1$. In addition, $\rho_{K}^{0}$ is defined as the solution that minimizes (14) subject to $\mathbf{x}(\rho, 0) \in \mathcal{D}_{K}$.

- Search over $K-1$ virtual LOS/NLOS partition scenarios: In the $k$ th search, $k=1,2, \ldots, K-1$, the UAV follows 
a similar procedure as that in Sections III-C2 and III-C3 for virtual LOS region $\tilde{\mathcal{D}}_{k}$ and virtual NLOS region $\mathcal{D}_{k}^{\mathrm{c}}$.

- Integration: During the whole search, the UAV keeps track of the minimum achievable cost $F_{\min }$ and the corresponding position $\hat{\mathrm{x}}$. When the algorithm terminates, $\hat{\mathbf{x}}$ gives the desired UAV position.

The entire search algorithm is summarized in Algorithm 1 . An example search trajectory under the $K=2$ model in the polar coordinate system is visualized in Fig. 4 (a), where the black curve represents the search trajectory and the dashed gray curves represent the contours of the cost function $F_{1}(\rho, \theta)$.

\section{E. Propagation Segment Detection}

As the parameters $a_{k}$ and $b_{k}$ and the statistics of $\xi_{k}$ in (2) are assumed known as discussed in Section II-B, the propagation segment can be determined using maximum likelihood detection. Let $h_{k}$ be the probability distribution function of the random variable $\xi_{k}$ in propagation segment $k$. Then, the maximum likelihood estimator of the propagation segment is given by

$$
\hat{k}=\underset{k=1,2, \ldots, K}{\operatorname{argmax}} h_{k}\left(y-b_{k}+a_{k} \log _{10} d(\mathbf{x})\right)
$$

where $y$ is the channel gain $G_{\mathrm{dB}}$ measured at the UAV location $\mathbf{x}$. In addition, if $\xi_{k}$ are zero mean Gaussian distributed with variance $\sigma_{k}^{2}$, then the detection rule can be simplified as

$$
\hat{k}=\underset{k=1,2, \ldots, K}{\operatorname{argmin}} \frac{1}{\sigma_{k}}\left|y-b_{k}+a_{k} \log _{10} d(\mathbf{x})\right| .
$$

Note that, in practice, the parameters $a_{k}$ and $b_{k}$ and the statistics of $\xi_{k}$ can be estimated in a separate training phase. Alternatively, the parameter estimation can be integrated in Step 1 in Algorithm 1 Specifically, the UAV moves along the BS-user axis (and, optionally, to a few random locations) to collect enough channel measurements. Then, a maximum likelihood estimation method can be performed to learn these parameters [26], [27].

\section{Global Optimality and Linear Search Length}

It turns out that Algorithm 1 can find the globally optimal solution to $\mathscr{P}$ for at least two types of cost functions $f$.

Condition 1. Assume that the cost function $f(x, y)$ in $\mathscr{P}$ satisfies $\frac{\partial^{2} f(x, y)}{\partial x \partial y}=0$,

$x \frac{\partial^{2} f(x, y)}{\partial x^{2}}+2 \frac{\partial f(x, y)}{\partial x} \geq 0, \quad y \frac{\partial^{2} f(x, y)}{\partial y^{2}}+2 \frac{\partial f(x, y)}{\partial y} \geq 0$

for every $x, y>0$.

It can be easily verified that the cost function (6) in the outage probability minimization example in Section II-C1 satisfies Condition 1.

Condition 2. Assume that the cost function $f(x, y)$ in $\mathscr{P}$ can be written as $\max \left\{f_{1}(x), f_{2}(y)\right\}$, where $f_{1}(x)$ and $f_{2}(y)$ are decreasing functions.
It is also clear that the cost function (7) in the rate maximization example in Section II-C2 satisfies Condition 2.

In addition, we discuss optimality for continuous-time algorithm trajectory $\mathbf{x}(t)$, which can be obtained from Algorithm 1 using infinitesimal step size $\delta=\mathcal{O}(d t)$ at each infinitesimal time slot $d t$. Specifically, the search trajectory $\mathbf{x}(t)$ in Algorithm 1 can be described by piece-wise continuous dynamic systems, where one replaces $\delta$ by $\kappa d t$ in (12) and $\gamma$ by $\kappa \bar{\gamma} d t$ in (13), in which $\kappa$ is a parameter that specifies the moving speed of the UAV. Accordingly, the continuous-time processes of the minimum cost $F_{\min }(t)$ and the position track record $\hat{\mathbf{x}}(t)$ are given by $F_{\min }(t)=$ $\operatorname{minimize}_{0 \leq \tau \leq t} f\left(g_{\mathrm{u}}\left(\mathbf{x}(\tau), g_{\mathrm{b}}(\mathbf{x}(\tau))\right.\right.$ and $\hat{\mathbf{x}}(t)=\mathbf{x}(\hat{\tau})$, respectively, where $\hat{\tau}=\arg \min _{0 \leq \tau \leq t} f\left(g_{\mathrm{u}}\left(\mathbf{x}(\tau), g_{\mathrm{b}}(\mathbf{x}(\tau))\right.\right.$.

\section{A. Global Optimality}

We first present the main optimality result as follows.

Theorem 1 (Global Optimality). Suppose that the cost function $f$ in $\mathscr{P}$ satisfies either Condition 1 or Condition 2. Then, $\hat{\mathbf{x}}(t)$ in Algorithm 1 converges to the globally optimal solution to $\mathscr{P}$ and $F_{\min }(t)$ converges to the minimum cost value in finite time.

Theorem 1 confirms that the globally optimal UAV position is attainable, even though the terrain topology could be arbitrarily complex.

The optimality result can be better understood from the polar coordinate system. From the definition of the fictitious segment cost functions $F_{k}(\rho, \theta)$ in 11 , problem $\mathscr{P}$ can be equivalently written as

$$
\mathscr{P}^{\prime}: \quad \operatorname{minimize}_{\rho \geq 0,-\pi \leq \theta \leq \pi} F(\rho, \theta) \triangleq \sum_{k=1}^{K} F_{k}(\rho, \theta) \mathbb{I}\left\{(\rho, \theta) \in \mathcal{P}_{k}\right\}
$$

where $\mathcal{P}_{k} \triangleq\left\{(\rho, \theta): \mathbf{x}(\rho, \theta) \in \mathcal{D}_{k}\right\}$ is the $k$ th propagation segment in the polar coordinate system. The optimal solution $\mathbf{x}^{\star}$ to $\mathscr{P}$ can be obtained as $\mathbf{x}^{\star}=\mathbf{x}\left(\rho^{\star}, \theta^{\star}\right)$, in which $\left(\rho^{\star}, \theta^{\star}\right)$ is the optimal solution to $\mathscr{P}^{\prime}$.

The following intermediate results provide some intuitions to understand Algorithm 1 and Theorem 1

Proposition 2 (Bounded Search Region). The optimal solution $\mathbf{x}^{\star}$ to $\mathscr{P}$ can be obtained as $\mathbf{x}\left(\rho^{\star}, \theta^{\star}\right)$, where $\left(\rho^{\star}, \theta^{\star}\right) \in \mathcal{P}$ and

$$
\mathcal{P}=\left\{(\rho, \theta): 0 \leq \rho \leq L \cos \theta,-\frac{\pi}{2} \leq \theta \leq \frac{\pi}{2}\right\}
$$

in which, $L \triangleq\left\|\mathbf{x}_{b}-\mathbf{x}_{u}\right\|$ is the horizontal distance from the $B S$ to the user.

\section{Proof. Please refer to Appendix A}

Proposition 2 justifies the first stopping criterion $\rho \geq L \cos \theta$ in Step 3) of Algorithm 11. An intuitive explanation is that when the UAV moves outside the region $\mathcal{P}$ in (17), one can always find a position in $\mathcal{P}$ that has an equal (or smaller) distances, respectively, to the BS and to the user in the same (or less obstructed) propagation segment $\mathcal{D}_{k}$, leading to an equal (or lower) cost to achieve. Therefore, the optimal UAV position is contained in $\mathcal{P}$. 
The following proposition justifies the second stopping criterion $\partial F_{k}(\rho, \theta) / \partial \rho \geq 0$.

Proposition 3 (Partial Optimality). Suppose that the cost function $f$ in $\mathscr{P}$ satisfies either Condition 1 or Condition 2. Then, $F_{k}(\rho, \theta)$ admits a unique local minimizer $\rho_{k}^{*}(\theta)$ over $\rho \geq 0$ for every fixed $\theta$, where $|\theta|<\pi / 2$.

Proof. Please refer to Appendix B

Due to the fact that $F_{k}(\rho, \theta)$ has a unique local minimum for every $\theta$, condition $\partial F_{k}(\rho, \theta) / \partial \rho \geq 0$ implies that $\rho \geq \rho_{k}^{*}(\theta)$. On the other hand, Step 3 ) of Algorithm 1 always increases $\rho$ (to be formally justified in Lemma 9 in Appendix $\mathrm{D}$. Therefore, it suffices to stop the search when the condition $\partial F_{k}(\rho, \theta) / \partial \rho \geq 0$ is met.

Based on Propositions 2 and 3 , the proof of Theorem 1 is derived in Appendix $\mathrm{C}$

\section{B. Maximum Length of the Algorithm Trajectory}

Here, we derive the worst-case trajectory length of Algorithm 1

Theorem 2 (Maximum Trajectory Length). The length of the search trajectory from Algorithm 11 is upper bounded by $(2.4 K-1.4) L$, where $L \triangleq\left\|\mathbf{x}_{b}-\mathbf{x}_{u}\right\|$ is the horizontal distance from the $B S$ to the user.

Proof. Please refer to Appendix D

Theorem 2 suggests that the algorithm must terminate in a finite number of steps given a positive step size $\delta>0$. The total number of steps scales as $\mathcal{O}(L / \delta)$. Surprisingly, the bound is linear in $L$ and does not depend on the actual terrain, i.e., the shapes of the propagation segments $\mathcal{D}_{k}$. As a benchmark, if one searches the optimal UAV position following the segment boundaries (a property from Proposition 11, the worst-case search length is not guaranteed to be linear in $L$, depending on the actual shapes of the boundaries (see, for example, Fig. 2(c)).

\section{Numerical Results}

Consider a dense urban area with buildings ranging from 545 meter height following a uniform distribution as illustrated in Fig. 2 (a). The user is represented by a red circle and the BS locates at the top right corner denoted by a blue triangle. The height of the BS is 45 meters, and the UAV moves at 50 meter above the ground. As a result, there is always LOS propagation between the UAV and the BS. Consider two propagation scenarios, LOS and NLOS, for the UAV-to-user link; this corresponds to many existing models in the literature for a fair comparison. Correspondingly, the parameters of the UAV-BS channel in (1) are chosen as $\left(\alpha_{0}, \log _{10} \beta_{0}\right)=$ $(2.08,-3.85)$; Rician fading with $20 \mathrm{~dB}$ K-factor is assumed according to the Rural Macro BS to UAV scenario in [23]. The parameters of the UAV-user channel in (5) are chosen as $\left(\alpha_{1}, \log _{10} \beta_{1}, \alpha_{2}, \log _{10} \beta_{2}\right)=(2.14,-3.69,3.03,-3.84)$; Rician fading with $9 \mathrm{~dB}$ K-factor is assumed for the LOS case

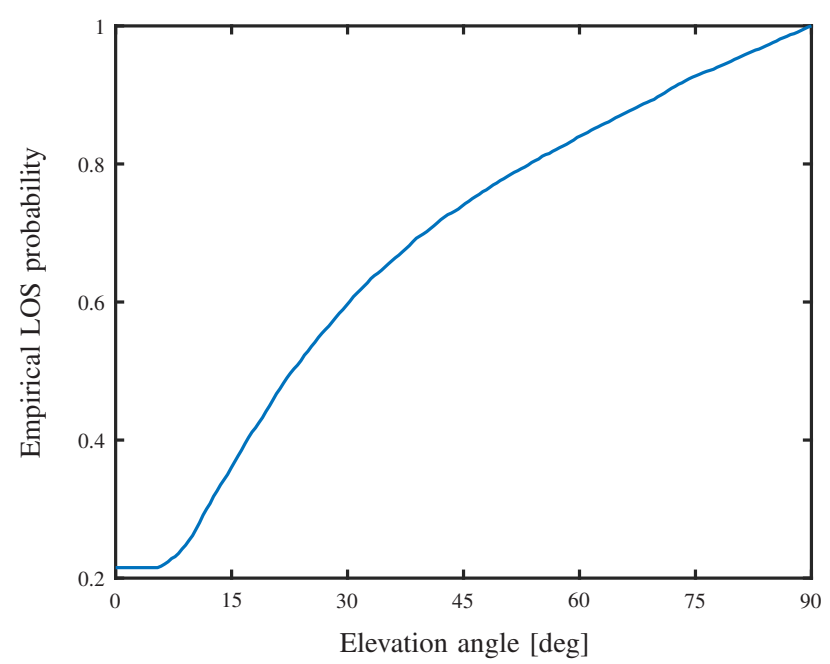

Figure 5. Empirical cumulative distribution function of the LOS probability in terms of the elevation angle estimated for the city topology shown in Fig. 2 (a). This is for evaluating the baseline scheme of Probabilistic Algorithm.

and Rayleigh fading is assumed for the NLOS case according to the Urban Micro BS to UAV scenario in [23].

We consider the example capacity maximization problem in Section II-C2 for optimal UAV positioning. The transmission powers are chosen as $P_{\mathrm{b}}=30 \mathrm{dBm}$ from the BS and $P_{\mathrm{d}}=36$ $\mathrm{dBm}$ from the UAV, and the noise power is $-80 \mathrm{dBm}$. The corresponding power map and end-to-end capacity map for every UAV position are illustrated in Fig. 2 (b) and (c).

In Fig. 4 (b), the green curves show the search path from Algorithm 11 The two curve branches correspond to the UAV searches in Step 2) and Step 4) in Algorithm 11 respectively. The other curves in Fig. 4 (b) represent the contour of the capacity map as in Fig. 2 (c). The optimal UAV position is found at the purple diamond.

\section{A. Throughput and Outage Probability for Single User Case}

We now evaluate the average throughput of UAV relay system for a single user located randomly and uniformly on the streets in Fig. 2 (a). The transmission powers are chosen as $P_{\mathrm{b}}=P_{\mathrm{d}}=33 \mathrm{dBm}$ for both the $\mathrm{BS}$ and the UAV, and the throughput is evaluated as the absolute value of (7). The proposed scheme places the UAV to the position obtained from Algorithm 1 under step size $\delta=5$ meters. We also consider the following baselines for UAV positioning:

- Probabilistic Algorithm [20]: First, obtain an empirical LOS distribution $f_{\operatorname{LOS}}(\varphi)=\mathbb{P}\{\operatorname{LOS}, \varphi\}$ as a function of the elevation angle $\varphi=\varphi\left(\mathbf{x}, \mathbf{x}_{\mathrm{u}}\right)$ from the user at $\left(\mathbf{x}_{\mathrm{u}}, 0\right)$ to the UAV at $\left(\mathbf{x}, H_{\mathrm{d}}\right)$, by uniformly and randomly dropping 10,000 users on the streets in Fig. 2 (a) and randomly picking UAV locations within the target area. The empirical distribution function $f_{\mathrm{LOS}}(\varphi)$ is shown in Fig. 5. Second, given each user position $\mathbf{x}_{\mathrm{u}}$, the UAV-user channel at UAV location $\mathbf{x}$ is computed as

$$
\begin{aligned}
g_{\mathrm{u}}\left(\mathbf{x}, \mathbf{x}_{\mathrm{u}}\right)= & f_{\mathrm{LOS}}(\varphi) \beta_{1}\left\|\mathbf{x}-\mathbf{x}_{\mathrm{u}}\right\|^{-\alpha_{1}} \\
& +\left(1-f_{\mathrm{LOS}}(\varphi)\right) \beta_{2}\left\|\mathbf{x}-\mathbf{x}_{\mathrm{u}}\right\|^{-\alpha_{2}} .
\end{aligned}
$$




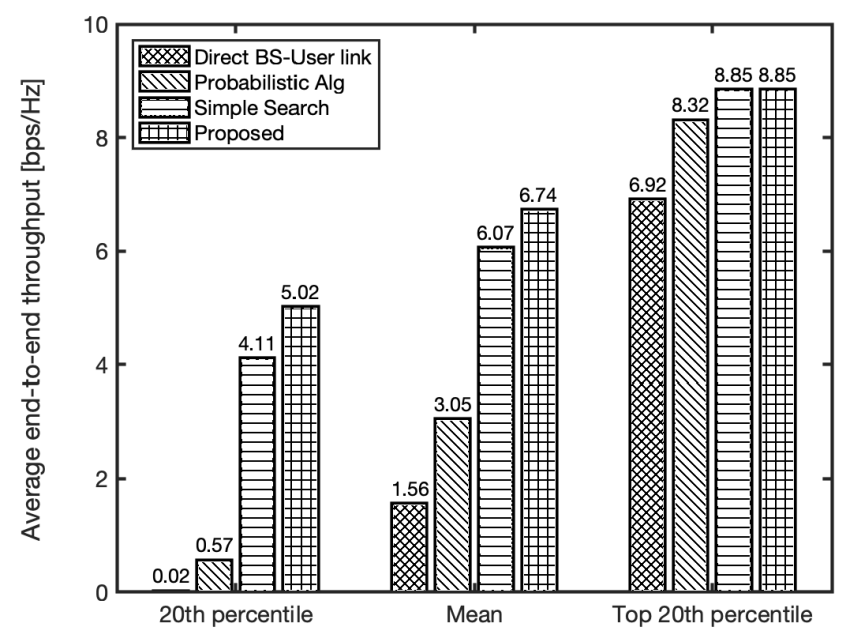

(a) Throughput of the three user categories

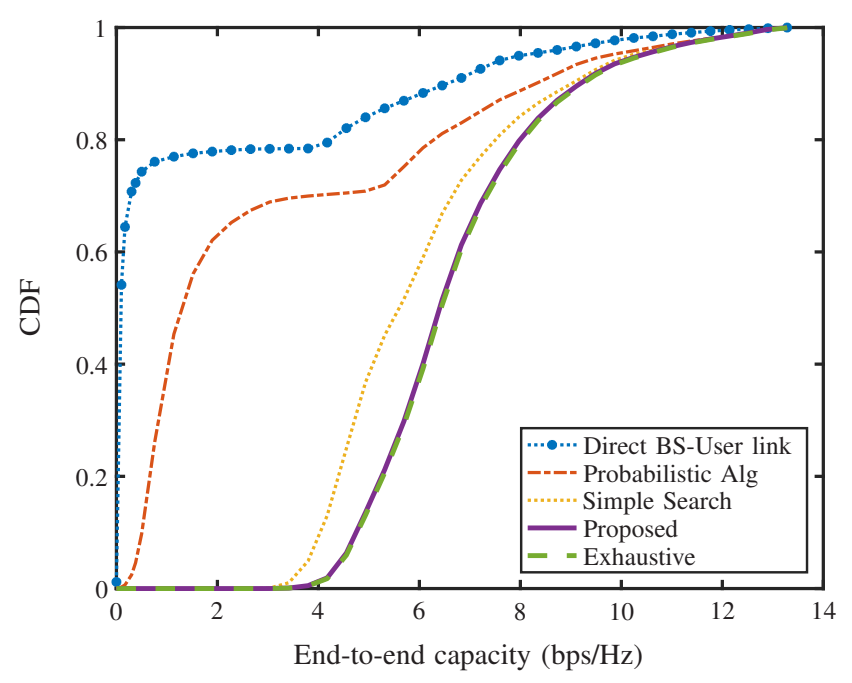

(b) $\mathrm{CDF}$ of the end-to-end throughput

Figure 6. Comparison of the average end-to-end throughput over different schemes.

The optimal UAV position is then obtained by solving $\mathscr{P}$ with $g_{\mathrm{u}}(\mathbf{x})=g_{\mathrm{u}}\left(\mathbf{x}, \mathbf{x}_{\mathrm{u}}\right)$ in 18 .

- Simple Search: Obtain the optimal UAV position by searching only on the BS-user axis (i.e., to implement only Step 1 of Algorithm 11.

- Exhaustive Search: We perform exhaustive search over the entire search region on equally-spaced grids with $\delta=5$ meter spacing. The grid point that maximizes the cost in (7) is chosen as the UAV position. Note that such a scheme is prohibited in practice and hence it is for benchmarking only.

The performance on direct BS-user transmission (without UAV relaying) is evaluated using the segmented channel model (5) by replacing $d_{\mathrm{u}}(\mathbf{x})$ by the BS-user distance and replacing $\mathbb{I}\left\{\mathbf{x} \in \mathcal{D}_{k}\right\}$ by the indicator of the BS-user link propagation condition.

Fig. 6 compares the average capacity of the Decode-andForward relay system discussed in Section II-C2 over 10,000

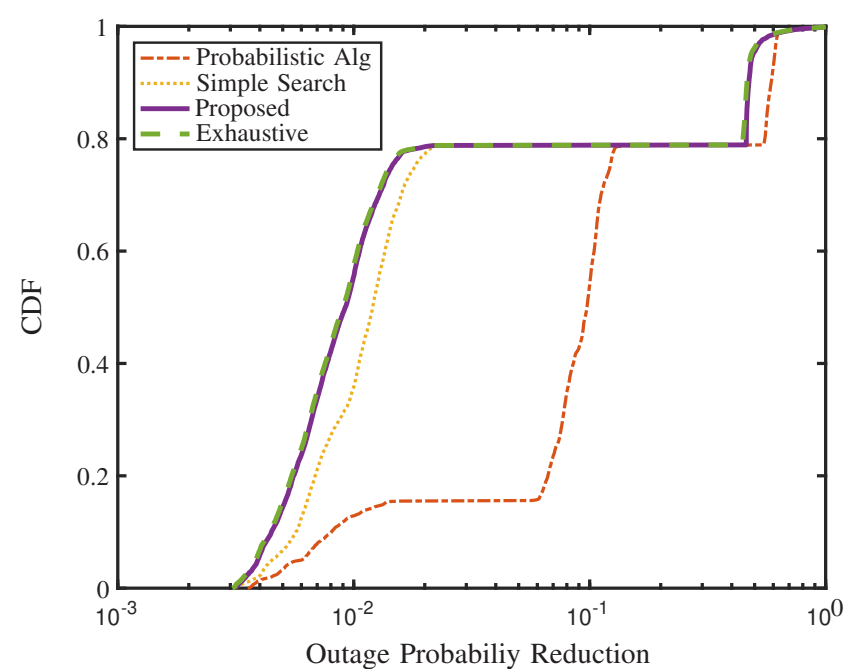

Figure 7. Outage probability reduction from the direct BS-user transmission scheme.

user positions in Fig. 2(a). The cell edge users are recognized as those within the 20th percentile of the throughput under direct BS-user transmission, and the cell center users are recognized as those in the top 20th percentile ${ }^{4}$ First, across all the three user categories shown in Fig. 6 (a), the proposed scheme with the optimal UAV placement achieves the highest throughput. In particular, it realizes more than $2 X$ throughput gain on average over an probabilistic approach. Second, the proposed scheme performs as well as the exhaustive search scheme as seen from the two overlapping cumulative distribution function (CDF) curves in Fig. 6 (b), which verify our theoretical results on optimality.

Fig. 7 compares the outage probability reduction from the direct BS-user transmission scheme using the Amplify-andForward relay system discussed in Section III-C1. Specifically, it is defined as $f\left(\mathbf{x}^{*}\right) / f_{0}$, where $f(\mathbf{x})$ is the cost function defined in (6) with $\mathrm{x}^{*}$ being the optimal UAV positions found by various schemes, and $f_{0}=1 /\left(P_{\mathrm{b}} g_{0}\left(\mathbf{x}_{\mathrm{u}}\right)\right)$, in which, $g_{0}\left(\mathbf{x}_{\mathbf{u}}\right)$ is the channel gain of the direct BS-user link. It is observed that the proposed scheme provides a significant outage probably reduction. In addition, it also confirms the globally optimality of the proposed scheme, when comparing its performance with the exhaustive search baseline.

\section{B. Clustered Multiuser Case}

We evaluate the proposed UAV placement strategy for the case of $N_{\mathrm{u}}=20$ users clustered around a hotspot with radius $r_{\mathrm{u}}$ meters. We implement a search trajectory from Algorithm 11 w.r.t. a virtual user located at $\mathbf{x}_{\mathrm{c}}$ and evaluate the sum data rate $\vec{f}(\mathbf{x})$ as discussed in Section II-C3. As a benchmark, the baseline scheme minimizes the average cost $\bar{f}(\mathbf{x})$ evaluated using the probabilistic model $g_{\mathrm{u}}\left(\mathbf{x}, \mathbf{x}_{\mathrm{u}}^{(i)}\right)$ in 18 for each user $i$. We drop 500 clusters uniformly and randomly in Fig. 2 (a), and evaluate the average throughput for the obstructed

\footnotetext{
${ }^{4}$ We found from our experiment that around $22 \%$ users have LOS condition on the BS-user link.
} 


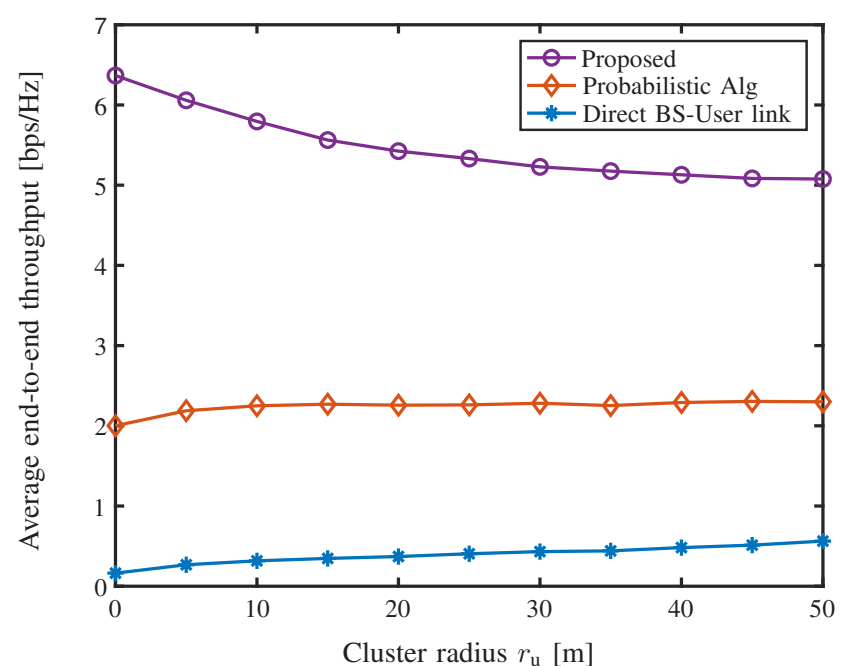

Figure 8. Average throughput for a cluster of $N_{\mathrm{u}}=20$ users distributed with radius at most $r_{\mathrm{u}}$ from the center of a hotspot.

clusters, of which the center position $\mathbf{x}_{\mathrm{c}}$ is in NLOS condition to the BS.

Fig. 8 shows the average throughput performance versus the radius $r_{\mathrm{u}}$ of the cluster. The case $r_{\mathrm{u}}=0$ refers to the single user scenario, and we know that Algorithm 1 achieves the globally optimal performance. When the cluster radius increases, the average throughput drops, because the proposed search algorithm is not optimized for the multiple users away from the cluster center. Nevertheless, the system still benefits from the proposed search strategy because the users nearby still share some degree of correlation in the propagation environment. As a result, the proposed strategy does also achieve significant performance gain over the probabilistic scheme.

\section{CONCLUSION}

This paper designed algorithms to search for the optimal UAV position for establishing the best wireless relay link between a BS and a user in a dense urban area, where global topological information, such as a 3D city map, is not available. The desired UAV position, constrained at a fixed height above the ground, was defined as the minima of a general cost function. A nested segmented propagation model was proposed to model the propagation from the UAV to the ground user that is probably blocked by obstacles. A search algorithm was developed and shown to find the globally optimal UAV position. In addition, the length of the search trajectory is upper bounded by a linear function of the diameter of the target area. Significant throughput gain was found when compared to other UAV positioning approaches or direct BSuser transmissions.

\section{APPENDIX A}

\section{PROOF OF PROPOSITION 2}

From the nested segmented propagation model (3)-(4), it is easy to verify that for any $\rho$ and $\theta$, the following holds

$$
F_{k}(\rho, \theta)<F_{k+1}(\rho, \theta)
$$

for $k=1,2, \ldots, K-1$.

The result of Proposition 2 can be proven by contradiction as follows.

First, suppose that solution $\mathbf{x}\left(\rho^{\prime}, \theta^{\prime}\right)$ satisfies $\frac{\pi}{2}<\left|\theta^{\prime}\right|<\pi$. Then, we must have $F_{1}\left(\rho^{\prime}, \theta^{\prime}\right) \leq F\left(\rho^{\prime}, \theta^{\prime}\right)$ from property 19 ) as $\mathcal{P}_{k}$ are mutually exclusive. Since there is LOS propagation when the UAV is on top of the outdoor user, i.e., $(0,0) \in \mathcal{P}_{1}$, we have

$$
F(0,0)=F_{1}(0,0)<F_{1}\left(\rho^{\prime}, \theta^{\prime}\right) \leq F\left(\rho^{\prime}, \theta^{\prime}\right)
$$

where, by moving from $(0,0)$ to $\left(\rho^{\prime}, \theta^{\prime}\right)$, the UAV position $\left(\rho^{\prime}, \theta^{\prime}\right)$ has longer distances to both the user and the BS. Thus, by contradiction, $\mathbf{x}\left(\rho^{\prime}, \theta^{\prime}\right)$ cannot be the optimal solution to $\mathscr{P}$, i.e., we must have $\left|\theta^{\prime}\right| \leq \frac{\pi}{2}$.

Second, suppose that the optimal solution $\mathbf{x}\left(\rho^{\prime}, \theta^{\prime}\right)$ satisfies $\rho^{\prime}>L \cos \theta^{\prime}$. Consider a different solution $\mathbf{x}\left(\rho^{\prime \prime}, \theta^{\prime}\right)$, where $\rho^{\prime \prime}=L \cos \theta^{\prime}-\left(\rho^{\prime}-L \cos \theta^{\prime}\right)$. From geometry, one can easily show that the UAV-BS distance $d_{\mathrm{b}}\left(\mathbf{x}\left(\rho^{\prime}, \theta^{\prime}\right)\right)=d_{\mathrm{b}}\left(\mathbf{x}\left(\rho^{\prime \prime}, \theta^{\prime}\right)\right)$ and the UAV-user distance $d_{\mathrm{u}}\left(\mathbf{x}\left(\rho^{\prime \prime}, \theta^{\prime}\right)\right)<d_{\mathrm{u}}\left(\mathbf{x}\left(\rho^{\prime}, \theta^{\prime}\right)\right)$. Suppose that $\left(\rho^{\prime}, \theta^{\prime}\right) \in \mathcal{P}_{k}$ and $\left(\rho^{\prime \prime}, \theta^{\prime}\right) \in \mathcal{P}_{j}$. From the nested segmented property (3)-(4), we must have $j \leq k$. From property (19), we have

$F\left(\rho^{\prime \prime}, \theta^{\prime}\right)=F_{j}\left(\rho^{\prime \prime}, \theta^{\prime}\right)<F_{k}\left(\rho^{\prime \prime}, \theta^{\prime}\right)<F_{k}\left(\rho^{\prime}, \theta^{\prime}\right)=F\left(\rho^{\prime}, \theta^{\prime}\right)$

which contradicts to the hypothesis that $\left(\rho^{\prime}, \theta^{\prime}\right)$ minimizes the objective function $F(\rho, \theta)$. This confirms that the optimal solution must satisfies $0 \leq \rho \leq L \cos \theta$.

\section{APPENDIX B \\ PROOF OF PROPOSITION 3}

\section{A. Proof under Condition I}

We first analyze the property of the composite function $f\left(\left(g_{\mathrm{u}} \circ d_{\mathrm{u}}\right)(\mathbf{z}),\left(g_{\mathrm{b}} \circ d_{\mathrm{b}}\right)(\mathbf{z})\right)$.

Lemma 1. Let $g_{i}\left(d_{i}\right)=\beta_{i} d_{i}^{-\alpha_{i}}, i=1,2$, and $\alpha>1$. Let $d_{i}(\mathbf{z}), i=1,2$, be convex functions in $\mathbf{z}$. Suppose that $f(x, y)$ satisfies Condition 1. Then, the composite function $f\left(\left(g_{1} \circ\right.\right.$ $\left.\left.d_{1}\right)(\mathbf{z}),\left(g_{2} \circ d_{2}\right)(\mathbf{z})\right)$ is strictly convex in $\mathbf{z}$.

Proof. Since $\partial f / \partial x<0$ and $\partial f / \partial y<0$, the first order partial derivative of $f\left(g_{1} \circ d_{1}, g_{2} \circ d_{2}\right)$ is given by

$$
\frac{\partial f}{\partial d_{i}}=\frac{\partial f}{\partial g_{i}} \frac{\partial g_{i}}{\partial d_{i}}=\frac{\partial f}{\partial g_{i}}\left(-\alpha_{i} \beta_{i}\right) d_{i}^{-\alpha_{i}-1}>0
$$

for $i=1,2$, and the second order partial derivative is given by

$$
\begin{aligned}
\frac{\partial^{2} f}{\partial d_{i}^{2}} & =\frac{\partial^{2} f}{\partial g_{i}^{2}} \frac{\partial g_{i}}{\partial d_{i}}\left(-\alpha_{i} \beta_{i}\right) d_{i}^{-\alpha_{i}-1}+\left(-\alpha_{i} \beta_{i}\right) \frac{\partial f}{\partial g_{i}} \frac{\partial}{\partial d_{i}} d_{i}^{-\alpha_{i}-1} \\
& =\alpha_{i}^{2} \beta_{i} d_{i}^{-\alpha_{i}-2}\left(\beta_{i} d_{i}^{-\alpha_{i}} \frac{\partial^{2} f}{\partial g_{i}^{2}}+\frac{\alpha_{i}+1}{\alpha_{i}} \frac{\partial f}{\partial g_{i}}\right) \\
& >\alpha_{i}^{2} \beta_{i} d_{i}^{-\alpha_{i}-2}\left(g_{i} \frac{\partial^{2} f}{\partial g_{i}^{2}}+2 \frac{\partial f}{\partial g_{i}}\right) \\
& \geq 0
\end{aligned}
$$

for $i=1,2$, where the first inequality is due to the fact that $\alpha_{i}>1$ and $\frac{\partial f}{\partial g_{i}}<0$. 
Define an operator $\nabla \triangleq\left[\frac{\partial}{\partial z_{1}} \frac{\partial}{\partial z_{2}} \cdots \frac{\partial}{\partial z_{m}}\right]^{\mathrm{T}}$, where $z_{i}$ is the $i$ th entry of a vector variable $\mathbf{z}$. From $\nabla f=\frac{\partial f}{\partial d_{1}} \nabla d_{1}+$ $\frac{\partial f}{\partial d_{2}} \nabla d_{2}$, the Hessian matrix of $f$ is given by

$$
\begin{aligned}
\nabla^{2} f= & \frac{\partial^{2} f}{\partial d_{1}^{2}} \nabla d_{1} \nabla d_{1}^{\mathrm{T}}+\frac{\partial f}{\partial d_{1}} \nabla^{2} d_{1} \\
& +\frac{\partial^{2} f}{\partial d_{2}^{2}} \nabla d_{2} \nabla d_{2}^{\mathrm{T}}+\frac{\partial f}{\partial d_{2}} \nabla^{2} d_{2} \succeq \mathbf{0}
\end{aligned}
$$

since $\nabla^{2} d_{i}(\mathbf{z}) \succeq \mathbf{0}$ due to the convexity of $d_{i}(\mathbf{z})$. Therefore, $f$ is strictly convex in $\mathbf{z}$ (as $d_{i}(\mathbf{z})$ are strictly convex).

From the polar representation of the UAV-user and UAV-BS distances

$$
\begin{aligned}
& d_{\mathrm{b}}(\mathbf{x}(\rho, \theta))=\sqrt{\rho^{2}+L^{2}-2 \rho L \cos \theta+\left(H_{\mathrm{d}}-H_{\mathrm{b}}\right)^{2}} \\
& d_{\mathrm{u}}(\mathbf{x}(\rho, \theta))=\sqrt{\rho^{2}+\left(H_{\mathrm{d}}-H_{\mathrm{u}}\right)^{2}}
\end{aligned}
$$

one can show that $d_{\mathbf{b}}(\mathbf{x}(\rho, \theta))$ and $d_{\mathfrak{u}}(\mathbf{x}(\rho, \theta))$ are strictly convex in $\rho$.

Then, using Lemma 1 and the definition of $F_{k}(\rho, \theta)$ in 11 , we can conclude that $F_{k}(\rho, \theta)$ is strictly convex in $\rho$, and therefore, $F_{k}(\rho, \theta)$ admits a unique local minima $\rho_{k}^{*}(\theta)$ in the bounded interval $\rho \in[0, L \cos \theta]$.

\section{B. Proof under Condition II}

Consider $F_{k}(\rho, \theta)=\max \left\{f_{1}\left(g_{\mathrm{u}}^{(k)}(\mathbf{x}(\rho, \theta)), f_{2}\left(g_{\mathrm{b}}(\mathbf{x}(\rho, \theta))\right\}\right.\right.$. From 201-(21), to increase $\rho$, we must have $d_{\mathrm{u}}$ increase and $d_{\mathrm{b}}$ decrease, and as a result, $f_{1}$ decreases and $f_{2}$ increases monotonically in $\rho \in[0, L \cos \theta]$. Therefore, these exists a unique local minimizer $\rho_{k}^{*}(\theta)$ in the closed interval $[0, L \cos \theta]$.

\section{APPENDIX C \\ PROOF OF THEOREM 1}

We first state the following lemma for the property of the fictitious segment cost functions $F_{k}(\rho, \theta)$ in 11 derived in the polar domain from the original objective $f$.

Lemma 2. It holds that

$$
\partial F_{k}(\rho, \theta) / \partial|\theta|>0
$$

for all $k$ and $\theta \neq 0$. In addition, for any $\theta^{\prime} \geq 0$, the following property holds

$$
\min _{\rho \geq 0} F_{k}\left(\rho, \theta^{\prime}\right) \leq \min _{j \geq k} \min _{\rho \geq 0, \theta^{\prime}<\theta \leq \frac{\pi}{2}} F_{j}(\rho, \theta)
$$

for $1 \leq k \leq K$. Similarly, for any $\theta^{\prime}<0$,

$$
\min _{\rho \geq 0} F_{k}\left(\rho, \theta^{\prime}\right) \leq \min _{j \geq k} \min _{\rho \geq 0,-\frac{\pi}{2} \leq \theta<\theta^{\prime}} F_{j}(\rho, \theta)
$$

Proof. We first note that $\frac{\partial}{\partial|\theta|} F_{k}(\rho, \theta)>0$ for all $k=$ $1,2, \ldots, K$, because increasing $|\theta|$ will increase the UAVBS distance $d_{\mathrm{b}}$ while the UAV-user distance $d_{\mathrm{u}}=\rho$ is not affected, and hence $g_{\mathbf{b}}(\mathbf{x}(\rho, \theta))$ is decreased. As the cost function $f(x, y)$ is increasing with $x$ and $y$, respectively, due to conditions I or II, the cost $F_{k}(\rho, \theta)$ increases as $|\theta|$ increases.
Consider that $\theta^{\prime} \geq 0$. For every $0 \leq \rho \leq L, \theta \geq \theta^{\prime}$, and $j \geq k$, we must have

$$
F_{k}\left(\rho, \theta^{\prime}\right) \leq F_{j}\left(\rho, \theta^{\prime}\right) \leq F_{j}(\rho, \theta) .
$$

As a result, $\min _{\rho \geq 0} F_{k}\left(\rho, \theta^{\prime}\right) \leq \min _{\rho \geq 0} F_{j}(\rho, \theta)$ for every $\theta>\theta^{\prime}$. Hence the result (23) is confirmed.

The case of 24 can be shown in a similar way.

Theorem 1 can be equivalently rewritten, in a more general setting, using the notions and conditions from the polar domain as follows:

Theorem 1A. Suppose that the set of segments $\left\{\mathcal{P}_{k}\right\}$ defined along with $\mathscr{P}^{\prime}$ satisfy the nested condition (4). In addition, assume that the globally optimal solution to $\mathscr{P}$ ' belongs to the bounded search region $\mathcal{P}$ defined in $(17)$, and the local minimizer $\rho_{k}^{*}(\theta)$ of $F_{k}(\rho, \theta)$ is unique for each $k$ and each $\theta,|\theta|<\frac{\pi}{2}$. Moreover, suppose that the functions $F_{k}(\rho, \theta)$ satisfy conditions (22)-(24). Then, the polar domain trajectory $(\hat{\rho}(t), \hat{\theta}(t))$ obtained from $\hat{\mathbf{x}}(t)$ following Algorithm 1 converges to the globally optimal solution to $\mathscr{P}^{\prime}$.

Note that it has been proven in Propositions 2 and 3 and Lemma 2 that the objective function $f$ (which satisfies either condition I or II) in the UAV positioning problem $\mathscr{P}$ (and, correspondingly, the functions $F_{k}$ in $\mathscr{P}^{\prime}$ ) satisfy the conditions in Theorem 1A. The remaining part of this section thus focuses on proving Theorem $1 \mathrm{~A}$.

\section{A. Optimality for the Two Segment Case}

When the algorithm terminates at $t=T$, it turns out that the cost value track record $F_{\min }(T)$ along with the algorithm trajectory satisfies $F_{\min }(T) \leq \operatorname{minimize}_{\rho \geq 0,(\rho, \theta) \in \mathcal{P}_{2}} F_{2}(\rho, \theta)$ because

$$
\begin{aligned}
F_{\min }(T) & \leq \underset{0 \leq \rho \leq L}{\operatorname{minimize}} \quad F(\rho, 0) \\
& =\underset{\rho \geq 0}{\operatorname{minimize}} \quad F(\rho, 0) \\
& \leq \underset{\rho \geq 0}{\operatorname{minimize}} \quad F_{2}(\rho, 0) \\
& \leq \underset{\rho \geq 0,(\rho, \theta) \in \mathcal{P}_{2}}{\operatorname{minimize}} F_{2}(\rho, \theta)
\end{aligned}
$$

where inequality (25) is due to Step 11 and 6 in Algorithm 1 and the fact that $F_{\min }(t)$ is a non-increasing process. Equality (26) is due to the condition in Theorem 1A (proven in Proposition 2), inequalities (27) and (28) are from conditions 23)-24) (proven in Lemma 2).

In fact, it also holds that

$$
F_{\min }(T) \leq \operatorname{minimize}_{\rho \geq 0,(\rho, \theta) \in \mathcal{P}_{1}} F_{1}(\rho, \theta)
$$

as follows.

Lemma 3 (Optimality for $K=2$ ). In the two segment case, the continuous trajectory $(\rho(t), \theta(t))$ passes through $\left(\rho^{*}, \theta^{*}\right)$ before it completes Step (3) in Algorithm 1 at $t=T_{1}$, i.e., there exists $t^{*} \leq T_{1}$, such that $\rho\left(t^{*}\right)=\rho^{*}$ and $\theta\left(t^{*}\right)=\theta^{*}$, where $\left(\rho^{*}, \theta^{*}\right)$ is the optimal solution to the following problem

$$
\underset{\rho \geq 0,(\rho, \theta) \in \mathcal{P}_{1}}{\operatorname{minmize}} F_{1}(\rho, \theta)
$$


where the minimum value equals to $F_{\min }\left(T_{1}\right)$.

Since $T_{1} \leq T$, we must have $F_{\min }(T) \leq F_{\min }\left(T_{1}\right)$ and the former one lower-bounds both the subproblems (28) and 29. Since $F_{\min }(t)$ represents the cost value track record along the trajectory $(\hat{\rho}(t), \hat{\theta}(t))$, we conclude that $(\hat{\rho}(T), \hat{\theta}(T))$ attains the globally optimal solution to $\mathscr{P}^{\prime}$ for the two segment case.

In the following two subsections, we first prove some preliminary properties of Algorithm 1 , and then, we use these properties to prove Lemma 3

\section{B. Preliminary Properties of Algorithm 1}

The following property states that, along the algorithm trajectory, the same cost $F_{1}(\rho(t), \theta(t))$ is achievable at some prior time $\tau \leq t$, even when the current step $(\rho(t), \theta(t))$ is not in the LOS region.

Lemma 4. For every point on the algorithm trajectory $(\rho(t), \theta(t)), 0 \leq t \leq T$, there exists $0 \leq \tau \leq t$, such that $(\rho(\tau), \theta(\tau)) \in \mathcal{P}_{1}$ and

$$
F(\rho(\tau), \theta(\tau))=F_{1}(\rho(\tau), \theta(\tau))=F_{1}(\rho(t), \theta(t)) .
$$

Proof. If $(\rho(t), \theta(t)) \in \mathcal{P}_{1}$, we have $F(\rho(t), \theta(t))=$ $F_{1}(\rho(t), \theta(t))$ and $\tau=t$. If $(\rho(t), \theta(t)) \in \mathcal{P}_{2}$, then the algorithm is in the loop of Step $3 \mathrm{p}$ ), where it follows the trajectory on the contour $F_{1}(\rho(t), \theta(t))=C$. To trace backward, there must be a $0 \leq \tau<t$, such that $F_{1}(\rho(\tau), \theta(\tau))=$ $F_{1}(\rho(t), \theta(t))$ and $(\rho(\tau), \theta(\tau)) \in \mathcal{P}_{1}$. Note that the initial point $\left(\rho_{1}^{0}, 0\right)$ from Step 1$)$ is in the LOS region $\mathcal{P}_{1}$ from the definition (8).

The following result shows that the algorithm trajectory always satisfies $\partial F_{1}(\rho, \theta(t)) / \partial \rho \leq 0$ until the algorithm terminates.

Lemma 5. Let $\rho_{1}^{*}(\theta)$ minimize $F_{1}(\rho, \theta)$ over all $\rho \geq 0$ with $\theta$ fixed. Then, the algorithm trajectory $(\rho(t), \theta(t))$ satisfies $\rho(t) \leq \rho_{1}^{*}(\theta(t))$ and $\partial F_{1}(\rho(t), \theta(t)) / \partial \rho \leq 0$ for all $t$ before the iteration completes Step 3). Moreover, $\partial F_{1}(\rho, \theta(t)) / \partial \rho \leq$ 0 for all $0 \leq \rho \leq \rho(t)$. The same result holds for the search trajectory in Step 4).

Proof. First, from the definition of $\rho_{1}^{0}$ in 14 and the nested segmented propagation property $(3)-(4)$, we have $\rho_{1}^{0} \leq \rho_{1}^{*}(0)$. This is because, we have $\mathbf{x}\left(\rho_{1}^{0}, 0\right) \in \mathcal{D}_{1}$ from $(14)$, and hence, all the points $(\rho, 0), 0 \leq \rho \leq \rho_{1}^{0}$, are in the LOS region. As a result, if $\rho_{1}^{0}>\rho_{1}^{*}(0)$, then $\left(\rho_{1}^{*}(0), 0\right)$ is also in the LOS region (satisfying the constraint $[15)$ ), which implies that $\rho_{1}^{*}(0)$ minimizes (14), yielding a contradiction. Therefore, from Step 1), we have the initial point $(\rho(0), \theta(0))$ satisfying $\theta(0)=0$ and $\rho(0)=\rho_{1}^{0} \leq \rho_{1}^{*}(0)=\rho_{1}^{*}(\theta(0))$.

Second, as the local minimizer $\rho_{1}^{*}(\theta)$ is unique from Proposition 3. we must have $\partial F_{1}(\rho, \theta) / \partial \rho<0$ for $\rho<\rho_{1}^{*}(\theta)$ and $\partial F_{1}(\rho, \theta) / \partial \rho>0$ for $\rho>\rho_{1}^{*}(\theta)$. (Note that there is no saddle point either, due to the strict convexity under Condition 1 and monotonicity of $f_{1}$ and $f_{2}$ under Condition 2.) Once $\partial F_{1}(\rho(t), \theta(t)) / \partial \rho \geq 0$, Step 3 is completed. As a result, it holds that $\rho(t) \leq \rho_{1}^{*}(\theta(t))$.

\section{Proof of Lemma 3}

It suffices to prove for the subproblem

$$
\mathscr{P}_{1+}^{\prime}: \quad F_{\min }\left(T_{1}\right) \leq \underset{\rho \geq 0,(\rho, \theta) \in \mathcal{P}_{1}^{+}}{\operatorname{minimize}} F_{1}(\rho, \theta)
$$

which is essentially solved by the iterations in Step 2) of Algorithm 1, where $\mathcal{P}_{1}^{+}=\left\{(\rho, \theta): 0 \leq \theta \leq \frac{\pi}{2},(\rho, \theta) \in \mathcal{P}_{1}\right\}$. Indeed, the counterpart subproblem over the constraint set $\mathcal{P}_{1}^{-}=\left\{(\rho, \theta):-\frac{\pi}{2} \leq \theta \leq 0,(\rho, \theta) \in \mathcal{P}_{1}\right\}$ is solved in a similar way by Step 4). If (30) holds, then it must also hold that

$$
F_{\min }\left(T_{1}\right) \leq \text { minimize }_{\rho \geq 0,(\rho, \theta) \in \mathcal{P}_{1}^{-}} F_{1}(\rho, \theta)
$$

which confirms the result of Lemma 3

Step A: We first show that the algorithm trajectory $(\rho(t), \theta(t))$ in the loop of Step 3 can only stop at $\theta(T) \geq \theta^{*}$, where $T \leq T_{1}$. Therefore, as the algorithm trajectory from Step 3 is continuous, we must have $\theta(t)=\theta^{*}$ for some $t \leq T$.

Lemma 6. The algorithm trajectory in Step 3) $(\rho(t), \theta(t))$ can only stop at $\theta(T)=\theta^{\prime} \geq \theta^{*}$ where $\left(\rho^{*}, \theta^{*}\right)$ is the optimal solution to $\mathscr{P}_{1+}^{\prime}$.

Proof. The result can be proven by contradiction. Suppose that Step 3 stops at $(\rho(T), \theta(T))$ where $\rho(T)=\rho^{\prime}$ and $\theta(T)=$ $\theta^{\prime}<\theta^{*}$. As Step 3) is completed, either one of the stopping criteria should have been triggered.

First, suppose that the condition $\partial F_{1}\left(\rho^{\prime}, \theta^{\prime}\right) / \partial \rho \geq 0$ was triggered. From Lemma 4, there exists $\tau \leq T$, such that $F_{1}(\rho(\tau), \theta(\tau))=F_{1}\left(\rho^{\prime}, \theta^{\prime}\right)$ and $(\rho(\tau), \theta(\tau)) \in \mathcal{P}_{1}^{+}$. From Lemma 5 and the condition in Theorem 1A that corresponds to Proposition 3. $\left(\rho^{\prime}, \theta^{\prime}\right)$ minimizes $F_{1}\left(\rho, \theta^{\prime}\right)$ over $\rho \geq 0$. As a result,

$$
\begin{aligned}
F_{1}(\rho(\tau), \theta(\tau)) & =F_{1}\left(\rho^{\prime}, \theta^{\prime}\right) \\
& =\underset{\rho \geq 0}{\operatorname{minimize}} F_{1}\left(\rho, \theta^{\prime}\right) \\
& \leq \min _{j \geq 1} \underset{\rho \geq 0, \theta^{\prime}<\theta \leq \frac{\pi}{2}}{\operatorname{minimize}} F_{j}(\rho, \theta) \\
& \leq \underset{\rho \geq 0, \theta^{\prime}<\theta \leq \frac{\pi}{2}}{\operatorname{minimize}} F_{1}(\rho, \theta) \\
& \leq \underset{\rho \geq 0, \theta^{\prime}<\theta \leq \frac{\pi}{2},(\rho, \theta) \in \mathcal{P}_{1}^{+}}{\operatorname{minimimize}} F_{1}(\rho, \theta) \\
& =F_{1}\left(\rho^{*}, \theta^{*}\right)
\end{aligned}
$$

where the first two inequalities are from the condition in Theorem 1A that corresponds to Lemma 2. The third equality is by the hypothesis $\theta^{*}>\theta^{\prime}$. However, this violates the assumption $\left(\rho^{*}, \theta^{*}\right)$ being the solution to subproblem $\mathscr{P}_{1+}^{\prime}$, since $(\rho(\tau), \theta(\tau)) \in \mathcal{P}_{1}^{+}$now yields a lower cost. By contradiction, the stopping criterion $\partial F_{1}\left(\rho^{\prime}, \theta^{\prime}\right) / \partial \rho \geq 0$ is not satisfied.

Second, suppose that the condition $\rho(T)=\rho^{\prime} \geq L \cos \theta^{\prime}$ is triggered. From the bounded search region condition in Theorem 1A (corresponding to Proposition 2) and from the hypothesis $\theta\left(T_{3}\right)=\theta^{\prime}<\theta^{*}$, we have

$$
\rho^{\prime} \geq L \cos \theta^{\prime}>L \cos \theta^{*} \geq \rho^{*} .
$$

From Lemma 5, $\rho_{1}^{*}\left(\theta^{\prime}\right)>\rho^{\prime}$ and $\partial F_{1}\left(\rho, \theta^{\prime}\right) / \partial \rho<0$ for $\rho^{\prime} \geq$ $\rho \geq \rho^{*}$. As a result,

$$
F_{1}\left(\rho^{\prime}, \theta^{\prime}\right)<F_{1}\left(\rho^{*}, \theta^{\prime}\right)<F_{1}\left(\rho^{*}, \theta^{*}\right)
$$


where the second inequality is from Lemma 2 .

From Lemma 4 there exists $\tau \leq T$, such that $(\rho(\tau), \theta(\tau)) \in$ $\mathcal{P}_{1}^{+}$and

$$
F_{1}(\rho(\tau), \theta(\tau))=F_{1}\left(\rho^{\prime}, \theta^{\prime}\right)<F_{1}\left(\rho^{*}, \theta^{*}\right)
$$

which contradicts to the hypothesis $\left(\rho^{*}, \theta^{*}\right)$ being the solution to subproblem $\mathscr{P}_{1+}^{\prime}$. Therefore, the stopping criterion $\rho\left(T_{3}\right)=$ $\rho^{\prime} \geq L \cos \theta^{\prime}$ is not satisfied either.

To conclude, since neither of the stopping criteria is triggered, by contradiction, the algorithm can only stop at $\theta(T) \geq$ $\theta^{*}$.

Step B: We then argue that when the trajectory reaches $\theta(t)=\theta^{*}$ for some $t \leq T$, it must hold that $(\rho(t), \theta(t)) \in \mathcal{P}_{1}^{+}$ and $\rho(t) \leq \rho^{*}$.

Lemma 7. The algorithm trajectory satisfies $\left(\rho\left(t_{1}\right), \theta\left(t_{1}\right)\right) \in$ $\mathcal{P}_{1}^{+}$, where $t_{1} \geq 0$ satisfies $\theta\left(t_{1}\right)=\theta^{*}$ and $\theta(t) \leq \theta^{*}$ for $t<t_{1}$.

Proof. We note that $\rho\left(t_{1}\right) \leq \rho^{*}$. This is because if $\rho\left(t_{1}\right)>\rho^{*}$, we must have $F_{1}\left(\rho\left(t_{1}\right), \theta^{*}\right)<F_{1}\left(\rho^{*}, \theta^{*}\right)$, since $\partial F_{1}\left(\rho, \theta^{*}\right) / \partial \rho<0$ for all $\rho\left(t_{1}\right)>\rho>\rho^{*}$ as from Lemma 5 As a result of Lemma 4, there exists $\tau \leq t_{1}$, such that

$$
F_{1}(\rho(\tau), \theta(\tau))=F_{1}\left(\rho\left(t_{1}\right), \theta^{*}\right)<F_{1}\left(\rho^{*}, \theta^{*}\right)
$$

and $(\rho(\tau), \theta(\tau)) \in \mathcal{P}_{1}^{+}$, which contradicts to the assumption that $\left(\rho^{*}, \theta^{*}\right)$ is the solution to 29 .

As $\left(\rho^{*}, \theta^{*}\right) \in \mathcal{P}_{1}^{+}$, from the nested segmented propagation property (3)-(4), we can conclude that $\left(\rho\left(t_{1}\right), \theta\left(t_{1}\right)\right) \in \mathcal{P}_{1}^{+}$.

Since $\left(\rho\left(t_{1}\right), \theta\left(t_{1}\right)\right) \in \mathcal{P}_{1}^{+}, \rho(t)$ will increase to $\rho^{*}$ following Step 3 ) in Algorithm 1 . This completes the proof that the algorithm trajectory passes through $\left(\rho\left(t_{2}\right), \theta\left(t_{2}\right)\right)=\left(\rho^{*}, \theta^{*}\right)$ at time $t_{2}$, where $t_{1} \leq t_{2} \leq T_{3}$.

Step C: Now, the algorithm iterate $(\rho(t), \theta(t))$ is at the stage of Step 33) in Algorithm 11, with $\theta(t)=\theta^{*}$ and $\rho(t) \leq \rho^{*}$. It must reach $\left(\rho^{*}, \theta^{*}\right)$ following Step 3 a $)$, where $\left(\rho^{*}, \theta^{*}\right)$ solves $\mathscr{P}_{1+}^{\prime}$.

With these, we confirm the results of Lemma 3

\section{Optimality for the K Segment Case}

We now extend Lemma 3 to the case of more than two segments.

Lemma 8 (Optimality after $k$ Loops). After completing the $k$ th loop of Steps 2) (4) in Algorithm 1 at time $t=T_{k}$, the following holds,

$$
F_{\min }\left(T_{k}\right) \leq \underset{\theta \geq 0,(\rho, \theta) \in \mathcal{P}_{k}}{\operatorname{minimize}} F_{k}(\rho, \theta)
$$

for all $k=1,2, \ldots, K-1$. Moreover, (34) holds for $k=K$ when the entire algorithm terminates.

Proof. For the $k$ th loop $(k \leq K-1)$ of Steps 2p - 4) in Algorithm 11. the iteration is equivalent to that in the two segment case, $K=2$. Specifically, the virtual propagation segment partition $\left(\tilde{\mathcal{P}}_{k}, \tilde{\mathcal{P}}_{k}^{\mathrm{c}}\right)$ in the $k$ th outer loop corresponds to the LOS-NLOS partition $\left(\mathcal{P}_{1}, \mathcal{P}_{2}\right)$ in the loop of Steps 2
- 4) for the $K=2$ case. Moreover, the function $F_{k}$ in the $k$ th outer loop corresponds to the function $F_{1}$ in the $K=2$ case. As a result, using Lemma 3, Steps 2) - 47 in the $k$ th loop equivalently solve

$$
\mathscr{P}_{k}^{\prime}: \underset{\theta \geq 0,(\rho, \theta) \in \widetilde{\mathcal{P}}_{k}}{\operatorname{minimize}} F_{k}(\rho, \theta)
$$

where $\tilde{\mathcal{P}}_{k} \triangleq \bigcup_{j=1}^{k} \mathcal{P}_{j}$ and $k \leq K-1$.

We denote the minimum value of 35 as $\tilde{F}_{\min , k}$, and the algorithm trajectory $(\rho(t), \theta(t))$ reaches the optimal solution $\left(\hat{\rho}^{(k)}, \hat{\theta}^{(k)}\right)$ at time $t_{k} \leq T_{k}$. Since the constraint set $\tilde{\mathcal{P}}_{k}$ in (35) contains the constraint set $\mathcal{P}_{k}$ in (34), it must hold that

$$
\tilde{F}_{\min , k} \leq \operatorname{minimize}_{\theta \geq 0,(\rho, \theta) \in \mathcal{P}_{k}} F_{k}(\rho, \theta) .
$$

Therefore, we still need to show $F_{\min }\left(T_{k}\right) \leq \tilde{F}_{\min , k}$.

With such a goal, the following two cases are examined:

(i) If $\left(\hat{\rho}^{(k)}, \hat{\theta}^{(k)}\right) \in \mathcal{P}_{k}$, then $F_{\min }\left(T_{k}\right) \leq F\left(\rho\left(t_{k}\right), \theta\left(t_{k}\right)\right)=$ $F_{k}\left(\rho\left(t_{k}\right), \theta\left(t_{k}\right)\right)=\tilde{F}_{\text {min,k }}$ due to the track record update $F_{\min }(t)$ from Step 3a).

(ii) If $\left(\hat{\rho}^{(k)}, \hat{\theta}^{(k)}\right) \notin \mathcal{P}_{k}$, then it holds that $\left(\hat{\rho}^{(k)}, \hat{\theta}^{(k)}\right) \in$ $\widetilde{\mathcal{P}}_{k} \backslash \mathcal{P}_{k}=\bigcup_{i<k} \mathcal{P}_{i}$. Without loss of generality (w.l.o.g.) assume that $\left(\hat{\rho}^{(k)}, \hat{\theta}^{(k)}\right) \in \mathcal{P}_{j}$ for some $j \leq k-1$. We thus have

$$
\begin{aligned}
\tilde{F}_{\min , k} & =F_{k}\left(\hat{\rho}^{(k)}, \hat{\theta}^{(k)}\right) \\
& \geq F_{j}\left(\hat{\rho}^{(k)}, \hat{\theta}^{(k)}\right) \\
& =F\left(\rho\left(t_{k}\right), \theta\left(t_{k}\right)\right) \\
& \geq F_{\min }\left(t_{k}\right) \\
& \geq F_{\min }\left(T_{k}\right)
\end{aligned}
$$

where the inequality on the first line is from conditions (23)-(24). The second line is due to the track record update $F_{\min }(t)$ from Step 3a). This shows that inequality (34) is also true for $k$.

As a result, we have shown

$$
F_{\min }\left(T_{k}\right) \leq \tilde{F}_{\min , k} \leq \operatorname{minimize}_{\theta \geq 0,(\rho, \theta) \in \mathcal{P}_{k}} F_{k}(\rho, \theta)
$$

for $k=1,2, \ldots, K-1$.

Finally, the last step in Algorithm 1 yields

$$
\begin{aligned}
F_{\min } & \leq F_{K}\left(\rho_{K}^{*}(0), 0\right) \\
& \leq \underset{\rho \geq 0,0<\theta \leq \frac{\pi}{2}}{\operatorname{minimize}} F_{K}(\rho, \theta) \\
& \leq \underset{\theta \geq 0,(\rho, \theta) \in \mathcal{P}_{K}}{\operatorname{minimize}} F_{K}(\rho, \theta)
\end{aligned}
$$

where the second inequality in 38 is from conditions (23)-24). The result of Lemma 8 is thus confirmed.

Theorem $1 \mathrm{~A}$ is a direct result from Lemma 8, since 34 holds for $k=1,2, \ldots, K$ and $F_{\min }(t)$ is non-increasing, which implies that $F_{\min }\left(T_{K}\right)$ is the global minimum value of $\mathscr{P}^{\prime}$ and $\left(\hat{\rho}\left(T_{K}\right), \hat{\theta}\left(T_{K}\right)\right)$ is the globally optimal solution to $\mathscr{P}^{\prime}$. 


\section{APPENDIX D \\ PROOF OF THEOREM 2}

An important property of Algorithm 1 is that the segment of search trajectory in Step 3 does not "turn back", and so does that in Step 4 .

Lemma 9 (Monotonicity). Step 3) in Algorithm 1 strictly and monotonically increases $\rho(t)$, and it also monotonically increases $|\theta(t)|$. Similar property holds in Step 4).

Proof. In Step 3 ) $), \rho(t)$ strictly and monotonically increases, while $\theta(t)$ keeps unchanged. In Step 3b), we have (omitting the higher order term)

$$
\begin{aligned}
\rho+\Delta \rho & =\left\|\mathbf{x}+\Delta \mathbf{x}-\mathbf{x}_{\mathrm{u}}\right\| \\
& =\sqrt{\left\|\mathbf{x}-\mathbf{x}_{\mathbf{u}}\right\|^{2}+2\left(\mathbf{x}-\mathbf{x}_{\mathrm{u}}\right)^{\mathrm{T}} \Delta \mathbf{x}+\|\Delta \mathbf{x}\|^{2}} \\
& =\rho\left(1+\frac{1}{\rho^{2}}\left(\mathbf{x}-\mathbf{x}_{\mathrm{u}}\right)^{\mathrm{T}} \Delta \mathbf{x}+o(\|\mathbf{x}\|)\right)
\end{aligned}
$$

and since $\left(\mathbf{x}-\mathbf{x}_{\mathbf{u}}\right) / \rho=\mathbf{M}(\theta) \mathbf{u}$ from 8 , we have

$$
\begin{aligned}
\Delta \rho= & \mathbf{u}^{\mathrm{T}} \mathbf{M}(\theta)^{\mathrm{T}} \Delta \mathbf{x} \\
= & \mathbf{u}^{\mathrm{T}} \mathbf{M}(\theta)^{\mathrm{T}} \mathbf{M}(\theta) \mathbf{u} \gamma \\
& \quad+\gamma \rho \mathbf{u}^{\mathrm{T}} \mathbf{M}(\theta)^{\mathrm{T}} \frac{d}{d \theta} \mathbf{M}(\theta) \mathbf{u}\left(-\frac{\partial F_{k}}{\partial \theta}\right)^{-1} \frac{\partial F_{k}}{\partial \rho}
\end{aligned}
$$

which equals to $\gamma$, being strictly positive. Therefore, Step 3 . strictly increases $\rho(t)$.

In addition, Step 33) moves on the contour of $F_{k}(\rho, \theta)=C$, whose dynamics is give by

$$
\frac{\partial F_{k}(\rho, \theta)}{\partial \rho} d \rho+\frac{\partial F_{k}(\rho, \theta)}{\partial \theta} d \theta=0
$$

in which $\partial F_{k}(\rho(t), \theta(t)) / \partial \rho \leq 0$ according to Lemma 5 (with a straight-forward generalization from $F_{1}$ to $F_{k}$ ) and $\partial F_{k}(\rho, \theta) / \partial|\theta|>0$ according to Lemma 2. As a result, Step 3.) monotonically increases $|\theta(t)|$.

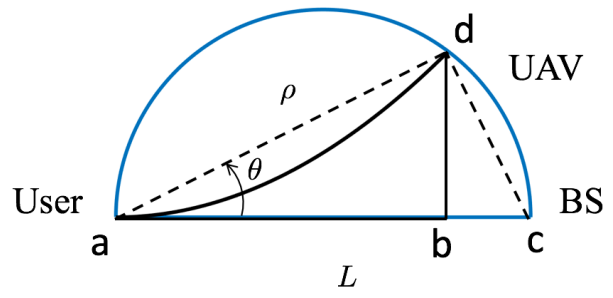

Figure 9. Search region and search trajectory, where point a denotes user, point $\mathbf{c}$ denotes the BS, and point $\mathbf{d}$ denotes UAV at the boundary of the search area.

We now show that the boundary of the search region $\mathcal{P}$ in (17) for the $\theta>0$ branch is given by a semi-circle as illustrated in Fig. 9 (the blue semi-circle). To see this, we first note that the boundary of $\mathcal{P}$ for $\theta>0$ is given by $(\rho \cos \theta, \rho \sin \theta)$, where $\rho=L \cos \theta$ and $0 \leq \theta \leq \pi / 2$. Therefore, $\overline{\mathbf{a d}}=L \cos \theta$, $\overline{\mathbf{a c}}=L, \overline{\mathbf{d c}}=\sqrt{\left(L \cos ^{2} \theta-L\right)^{2}+(L \cos \theta \sin \theta-0)^{2}}$, which yields $(\overline{\mathbf{a d}})^{2}+(\overline{\mathbf{d c}})^{2}=(\overline{\mathbf{a c}})^{2}$ and hence point $\mathbf{d}$ is on the semi-circle with the diameter given by line segment $\overline{\mathbf{a c}}$.
From the monotone property in Lemma 9, the search trajectory is a convex curve starting from point a at the user and ending at the semi-circle, e.g., point $\mathbf{d}$. Note that the length of any curve ad is less than $\overline{\mathbf{a b}}+\overline{\mathbf{b d}}=L \cos ^{2} \theta+L \cos \theta \sin \theta$, where the maximum value over $0 \leq \theta<\pi / 2$ can be numerically evaluated to be roughly $1.2 \mathrm{~L}$.

Algorithm 1 consists of the search on the BS-user axis with maximum length $L$, and $K-1$ loops for the off-BS-user axis searches, where each loop consists of searches on the left and right branches each with maximum length $1.2 L$. As a result, the total length of the search trajectory is upper bounded by $(2.4 K-1.4) L$.

\section{REFERENCES}

[1] Y. Zeng, R. Zhang, and T. J. Lim, "Wireless communications with unmanned aerial vehicles: opportunities and challenges," IEEE Commun. Mag., vol. 54, no. 5, pp. 36-42, 2016.

[2] M. Mozaffari, W. Saad, M. Bennis, and M. Debbah, "Unmanned aerial vehicle with underlaid device-to-device communications: Performance and tradeoffs," IEEE Trans. Wireless Commun., vol. 15, no. 6, pp. 39493963, 2016.

[3] Z. Xiao, P. Xia, and X.-G. Xia, "Enabling UAV cellular with millimeterwave communication: Potentials and approaches," IEEE Commun. Mag., vol. 54, no. 5, pp. 66-73, 2016.

[4] B. Van Der Bergh, A. Chiumento, and S. Pollin, "LTE in the sky: trading off propagation benefits with interference costs for aerial nodes," IEEE Commun. Mag., vol. 54, no. 5, pp. 44-50, 2016.

[5] A. Merwaday and I. Guvenc, "UAV assisted heterogeneous networks for public safety communications," in Proc. Wireless Commun. and Networking Conf. Workshops, 2015, pp. 329-334.

[6] D.-T. Ho, P. Sujit, T. A. Johansen, and J. B. De Sousa, "Performance evaluation of cooperative relay and particle swarm optimization path planning for UAV and wireless sensor network," in Proc. IEEE Globecom Workshops, 2013, pp. 1403-1408.

[7] V. Sharma, M. Bennis, and R. Kumar, "UAV-assisted heterogeneous networks for capacity enhancement," IEEE Commun. Lett., vol. 20, no. 6 , pp. 1207-1210, 2016.

[8] O. Jian, Z. Yi, L. Min, and L. Jia, "Optimization of beamforming and path planning for UAV-assisted wireless relay networks," Chinese Journal of Aeronautics, vol. 27, no. 2, pp. 313-320, 2014.

[9] Y. Zeng and R. Zhang, "Energy-efficient UAV communication with trajectory optimization," IEEE Trans. Wireless Commun., vol. 16, no. 6, pp. 3747-3760, 2017.

[10] Y. Jin, Y. D. Zhang, and B. K. Chalise, "Joint optimization of relay position and power allocation in cooperative broadcast wireless networks," in Proc. IEEE Int. Conf. Acoustics, Speech, and Signal Processing, 2012, pp. 2493-2496.

[11] D. H. Choi, B. H. Jung, and D. K. Sung, "Low-complexity maneuvering control of a UAV-based relay without location information of mobile ground nodes," in Proc. IEEE Symposium on Computers and Commun., 2014, pp. 1-6.

[12] A. Chamseddine, G. Charland-Arcand, O. Akhrif, S. Gagné, F. Gagnon, and D. Couillard, "Optimal position seeking for unmanned aerial vehicle communication relay using only signal strength and angle of arrival," in IEEE Conf. on Decision and Control, Dec 2014, pp. 976-981.

[13] F. Jiang and A. L. Swindlehurst, "Optimization of UAV heading for the ground-to-air uplink," IEEE J. Sel. Areas Commun., vol. 30, no. 5, pp. 993-1005, 2012.

[14] M. M. Azari, F. Rosas, K.-C. Chen, and S. Pollin, "Ultra reliable UAV communication using altitude and cooperation diversity," IEEE Trans. Commun., vol. 66, no. 1, pp. 330-344, 2018.

[15] S. ur Rahman and Y.-Z. Cho, "UAV positioning for throughput maximization," EURASIP J. on Wireless Commun. and Networking, vol. 2018, no. 1, p. 31, 2018.

[16] J. Lyu, Y. Zeng, R. Zhang, and T. J. Lim, "Placement optimization of UAV-mounted mobile base stations," IEEE Commun. Lett., vol. 21, no. 3, pp. 604-607, 2017.

[17] A. Al-Hourani, S. Kandeepan, and A. Jamalipour, "Modeling air-toground path loss for low altitude platforms in urban environments," in Proc. IEEE Global Telecomm. Conf., 2014, pp. 2898-2904. 
[18] M. Mozaffari, W. Saad, M. Bennis, and M. Debbah, "Drone small cells in the clouds: Design, deployment and performance analysis," in Proc. IEEE Global Telecomm. Conf., 2015, pp. 1-6.

[19] A. Hourani, K. Sithamparanathan, and S. Lardner, "Optimal LAP altitude for maximum coverage," IEEE Commun. Lett., no. 99, pp. 1-4, 2014.

[20] M. Mozaffari, W. Saad, M. Bennis, and M. Debbah, "Optimal transport theory for power-efficient deployment of unmanned aerial vehicles," in Proc. IEEE Int. Conf. Commun., Kuala Lumpur, Malaysia, May 2016, pp. 1-6.

[21] optimal wireless coverage," IEEE Commun. Lett., vol. 20, no. 8, 2016.

[22] Q. Feng, J. McGeehan, E. K. Tameh, and A. R. Nix, "Path loss models for air-to-ground radio channels in urban environments," in Proc. IEEE Semiannual Veh. Technol. Conf., vol. 6, 2006, pp. 2901-2905.

[23] "Study on enhanced LTE support for aerial vehicles (release 15)," 3GPP TR 36.777, Tech. Rep., 2017. [Online]. Available: http://www.3gpp.org

[24] J. Chen and D. Gesbert, "Optimal positioning of flying relays for wireless networks: A LOS map approach," in Proc. IEEE Int. Conf. Commun., Paris, France, May 2017.

[25] T. Bai and R. W. Heath, "Coverage and rate analysis for millimeter-wave cellular networks," IEEE Trans. Wireless Commun., vol. 14, no. 2, pp. 1100-1114, 2015.

[26] J. Chen, U. Yatnalli, and D. Gesbert, "Learning radio maps for UAVaided wireless networks: A segmented regression approach," in Proc. IEEE Int. Conf. Commun., Paris, France, May 2017.

[27] J. Chen, O. Esrafilian, D. Gesbert, and U. Mitra, "Efficient algorithms for air-to-ground channel reconstruction in UAV-aided communications," in Proc. IEEE Global Telecomm. Conf., Dec. 2017, Wi-UAV workshop.

[28] R. U. Nabar, H. Bolcskei, and F. W. Kneubuhler, "Fading relay channels: Performance limits and space-time signal design," IEEE J. Sel. Areas Commun., vol. 22, no. 6, pp. 1099-1109, 2004.

[29] J. N. Laneman, D. N. Tse, and G. W. Wornell, "Cooperative diversity in wireless networks: Efficient protocols and outage behavior," IEEE Trans. Inf. Theory, vol. 50, no. 12, pp. 3062-3080, 2004.

[30] T. Wang, A. Cano, G. B. Giannakis, and J. N. Laneman, "Highperformance cooperative demodulation with decode-and-forward relays," IEEE Trans. Commun., vol. 55, no. 7, pp. 1427-1438, 2007. 